Analysis of dynamics, stability, and flow fields' structure of an accelerated hydrodynamic discontinuity with interfacial mass flux by a general matrix method

D. V. Ilyin, Y. Fukumoto, W. A. Goddard, and S. I. Abarzhi

Citation: Physics of Plasmas 25, 112105 (2018); doi: 10.1063/1.5008648

View online: https://doi.org/10.1063/1.5008648

View Table of Contents: http://aip.scitation.org/toc/php/25/11

Published by the American Institute of Physics

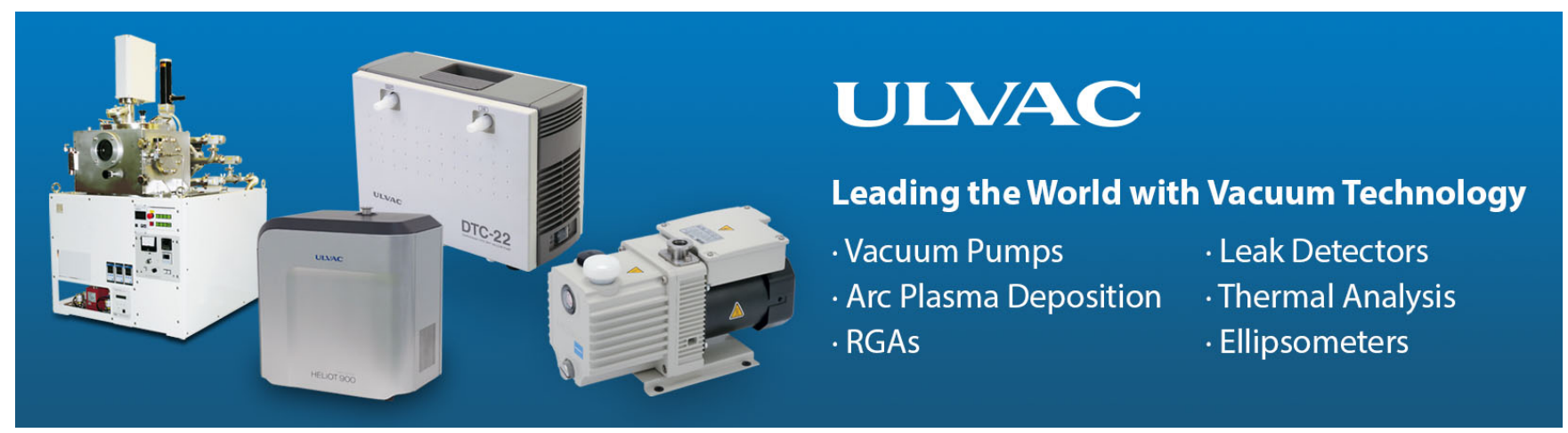




\title{
Analysis of dynamics, stability, and flow fields' structure of an accelerated hydrodynamic discontinuity with interfacial mass flux by a general matrix method
}

\author{
D. V. Ilyin, ${ }^{1}$ Y. Fukumoto, ${ }^{2}$ W. A. Goddard III, ${ }^{1}$ and S. I. Abarzhi ${ }^{3, a)}$ \\ ${ }^{1}$ California Institute of Technology, Pasadena, California 91125, USA \\ ${ }^{2}$ Kyushu University, Fukuoka, Fukuoka Prefecture 819-0395, Japan \\ ${ }^{3}$ The University of Western Australia, Perth, Washington 6009, Australia
}

(Received 9 October 2017; accepted 19 October 2018; published online 8 November 2018)

We develop a general matrix method to analyze from a far field the dynamics of an accelerated interface between incompressible ideal fluids of different densities with interfacial mass flux and with negligible density variations and stratification. We rigorously solve the linearized boundary value problem for the dynamics conserving mass, momentum, and energy in the bulk and at the interface. We find a new hydrodynamic instability that develops only when the acceleration magnitude exceeds a threshold. This critical threshold value depends on the magnitudes of the steady velocities of the fluids, the ratio of their densities, and the wavelength of the initial perturbation. The flow has potential velocity fields in the fluid bulk and is shear-free at the interface. The interface stability is set by the interplay of inertia and gravity. For weak acceleration, inertial effects dominate, and the flow fields experience stable oscillations. For strong acceleration, gravity effects dominate, and the dynamics is unstable. For strong accelerations, this new hydrodynamic instability grows faster than accelerated Landau-Darrieus and Rayleigh-Taylor instabilities. For given values of the fluids' densities and their steady bulk velocities, and for a given magnitude of acceleration, we find the critical and maximum values of the initial perturbation wavelength at which this new instability can be stabilized and at which its growth is the fastest. The quantitative, qualitative, and formal properties of the accelerated conservative dynamics depart from those of accelerated Landau-Darrieus and Rayleigh-Taylor dynamics. New diagnostic benchmarks are identified for experiments and simulations of unstable interfaces. Published by AIP Publishing.

https://doi.org/10.1063/1.5008648

\section{INTRODUCTION}

Hydrodynamic instabilities and interfacial mixing control a broad range of processes in nature and technology in plasmas, fluids, and materials. These processes occur at astrophysical and atomic scales, in high and low energy density regimes. ${ }^{1-3}$ Examples include supernovae explosions, planetary convection, inertial confinement fusion, lightmaterial interaction, material transformation, and supercritical fluids. $^{4-10}$ In realistic environments, the material transport is often characterized by sharp and rapid changes in the flow fields and by relatively small effects of dissipation and diffusion. At macroscopic (i.e., continuous) scales, these conditions lead to the formation of discontinuities (referred to as fronts or interfaces) between the flow nonuniformities (phases). ${ }^{11-13}$ Here, we consider from a far field the evolution of a hydrodynamic discontinuity separating fluids of different densities. The fluids are incompressible and ideal. There is a mass flow across the discontinuity. ${ }^{3} \mathrm{We}$ study the dynamics conserving mass, momentum, and energy in the fluids' bulk and at the interface. We find that the accelerated conservative dynamics has potential velocity fields in the fluid bulk and is shear-free at the interface and that it is

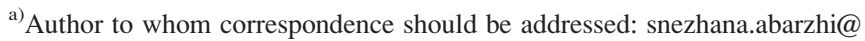
gmail.com
}

unstable only when the acceleration value exceeds a threshold. This hydrodynamic instability has the new quantitative, qualitative, and formal properties that unambiguously differentiate it from those of other fluid instabilities.

For a far field observer, two types of hydrodynamic discontinuities are ordinarily considered-a front (with zero mass transport across it) and an interface (with non-zero mass transport). ${ }^{3}$ Their dynamics is analyzed at length and time scales which are substantially greater than the characteristic scales induced by stabilizing effects (i.e., dissipation and diffusion) and is obtained as a solution of a boundary value problem at a freely evolving discontinuity. ${ }^{1-4}$ This approach is applicable in a broad parameter regime, and serves to identify reliable diagnostic benchmarks for experiments.

In the presence of acceleration, the front separating fluids of different densities may be subject to the RayleighTaylor instability (RTI). ${ }^{1,4,1-13}$ A rigorous group theory approach has been developed to solve the boundary value problem and to describe Rayleigh-Taylor (RT) flows which account for their non-local, anisotropic, and heterogeneous dynamics. $^{4,11,31}$ This approach captures the fundamental properties of the RTI and RT mixing (such as the multi-scale RT dynamics, and the order in RT mixing) and explains experimental observations. ${ }^{4,11,31}$

Here, we study the effect of acceleration on dynamics of interfaces. An interface is broadly defined as a phase 
boundary at which the fluid's thermodynamic properties change, and/or at which one fluid is transformed into the other. ${ }^{1-4}$ The evolution of an accelerated interface with the interfacial mass flux is a long-standing problem with a broad range of applications. ${ }^{1}$ It is studied in plasmas (stability of ablation fronts in inertial confinement fusion), astrophysics (thermonuclear flashes on surface of compact stars), materials science (material melting and evaporation), and industry (scramjets). ${ }^{1,-19}$ A classical theoretical framework for the problem has been developed by Landau. ${ }^{20}$ By balancing at the interface the fluxes of mass and momentum and by implementing a special condition for the perturbed mass flux, Landau has found that the interface is unconditionally unstable. This is known as the Landau-Darrieus instability (LDI). ${ }^{20}$

To connect the classical framework ${ }^{20}$ to realistic environments, several theoretical approaches have been developed. In high energy density plasmas (HEDP), significant departures have been detected between ablative Rayleigh-Taylor (RT) and Landau-Darrieus (LD) instabilities in gravity field, as well as between ablative Richtmyer-Meshkov (RM) and LD instabilities. $^{14-16,25-28}$ In reactive and super-critical fluids, the interfacial dynamics has been found to be stabilized by dissipation, diffusion, and finite thickness of the interface. ${ }^{10,17,21-23}$ Significant progress has been achieved toward understanding the nonlinear stages of the LDI and modeling turbulent combustion. ${ }^{17}$ For the conservative dynamics in strongly compressible fluids, the theoretical framework has been elaborated to study the stability of shocks. ${ }^{32,33}$ For incompressible fluids, it has been recently found that the interface stability is highly sensitive to energy fluctuations produced by the perturbed interface. $^{24,34}$

These theories and models successfully expanded the classical framework ${ }^{20,23}$ and explained experiments. ${ }^{15-17,21-23,25-28,31}$ Some fundamental challenges still remain. For instance: (i) The general theoretical framework ${ }^{20}$ describes the evolution of a phase boundary and is relevant to a range of phenomena far beyond the processes with gradually changing flow fields. We are yet to understand whether the interface is stable when the flow quantities experience sharp changes and when the effects of dissipation and diffusion are negligible. (ii) One usually observes the flow evolution from a far field and at time scales and length scales that are substantially greater than the characteristic scales induced by, e.g., diffusion, dissipation, finite thickness, and energy fluctuations. We need to better comprehend what the qualitative influence is of the interfacial transport at microscopic scales on flow fields at macroscopic scales in the bulk. (iii) There are still unresolved fundamental aspects in the theory of hydrodynamic discontinuities. In particular, we further need to quantify what the flow sensitivity is to the boundary conditions at the interface.

This knowledge is necessary to identify the mechanisms of stabilization of an accelerated phase boundary, to apply it in inertial confinement fusion (ICF) and supernova modeling, and to further improve the diagnostics of unstable interfacial dynamics in plasmas, fluids, and materials. ${ }^{1-34}$

In this work, we analyze from a far field the dynamics of an accelerated interface between incompressible ideal fluids of different densities with interfacial mass flux and negligible density variations and stratification. A two-dimensional periodic spatially extended flow is considered. We develop and apply a general matrix method to rigorously solve the linearized boundary value problem. We find a new hydrodynamic instability that develops only when the acceleration magnitude $g$ exceeds a critical threshold value $g_{c r}=k V_{h}^{2}\left(\rho_{h} / \rho_{l}\right)\left(\rho_{h}-\rho_{l}\right) /\left(\rho_{h}+\rho_{l}\right)$. The acceleration $\mathbf{g}$ is directed from the heavy to the light fluid, $g=|\mathbf{g}|$; the subscript $h(l)$ marks the heavy (light) fluid; the fluid densities are $\rho_{h(l)}$; the magnitudes of the steady velocities of the fluids are $V_{h(l)}$ with $\rho_{h} V_{h}=\rho_{l} V_{l}$; the wavevector is $k=2 \pi / \lambda$; and the wavelength of the initial perturbation of the interface is $\lambda$. The dynamics conserves mass, momentum, and energy in the fluid bulk and at the interface. The flow has potential velocity fields in the fluid bulk and is shear-free at the interface. The interface stability is set by the interplay of inertia and gravity. For weak acceleration, $g<g_{c r}$, the inertial effects dominate, and the flow fields experience stable oscillations. For strong acceleration, $g>g_{c r}$, the gravity effects dominate, and the dynamics is unstable. For strong accelerations, this new hydrodynamic instability grows faster than accelerated LDI and RTI. For given values of the fluids' densities and steady velocities, and for a given magnitudes of gravity, we find the critical and maximum values of the initial perturbation wavelength at which this new instability can be stabilized and at which its growth is the fastest. We find that the quantitative, qualitative, and formal properties of the accelerated conservative dynamics depart from those of the accelerated Landau-Darrieus and the Rayleigh-Taylor dynamics. Based on the obtained theoretical results, we identify new diagnostic benchmarks for experiments and simulations studying unstable interfaces, Tables I-III, Figs. 1-12. 3,4,6-34,36

\section{METHODS-GOVERNING EQUATIONS, LINEARIZED DYNAMICS, AND FUNDAMENTAL SOLUTIONS}

\section{A. Governing equations}

The dynamics of ideal fluids is governed by the conservation of mass, momentum, and energy as

$$
\begin{aligned}
& \partial \rho / \partial t+\partial \rho v_{i} / \partial x_{i}=0 \\
& \partial \rho v_{i} / \partial t+\sum_{j=1}^{3} \partial \rho v_{i} v_{j} / \partial x_{i}+\partial P / \partial x_{i}=0 \\
& \partial E / \partial t+\partial(E+P) v_{i} / \partial x_{i}=0
\end{aligned}
$$

where $x_{i}$ are the spatial coordinates with $\left(x_{1}, x_{2}, x_{3}\right)$ $=(x, y, z), t$ is time, $(\rho, \mathbf{v}, P, E)$ are the fields of density $\rho$, velocity $\mathbf{v}$, pressure $P$ and energy $E=\rho\left(e+\mathbf{v}^{2} / 2\right)$, and $e$ is specific internal energy in its physics definition. ${ }^{3}$ That is, the specific internal energy refers to energy per unit mass that is contained within a system, excluding the kinetic energy of motion of the system as a whole and the potential energy of the system as a whole due to the external force fields. ${ }^{3,24}$ The inertial frame of reference refers to the frame of reference moving with a constant velocity $\tilde{\mathbf{V}}_{0}=\left(0,0, \tilde{V}_{0}\right)$.,34

We introduce a continuous local scalar function $\theta(x, y, z, t)$, whose derivatives $\dot{\theta}$ and $\nabla \theta$ exist (the dot marks 
TABLE I. The growth-rates of the new conservative instability $\omega_{C S G}$, the Landau-Darrieus instability $\omega_{L D G}$, and the Rayleigh-Taylor $\omega_{R T}$ instability in a gravity field $G, G \in(0,+\infty)$, for incompressible ideal fluids with the density ratio $R, R \in\left(1^{+},+\infty\right)$. The values are $\omega_{C S G}=i \sqrt{R} \sqrt{1-G / G_{c r}}$, $\omega_{L D G}=\left(-R+\sqrt{\left(R^{3}+R^{2}-R\right)+G\left(R^{2}-1\right)}\right) /(1+R), \omega_{R T}=\sqrt{G(R-1) /(1+R)}$ with $G_{c r}=R(R-1) /(R+1), G^{*}=\left(R^{2}-1\right) / 4$.

\begin{tabular}{lccccc}
\hline \hline & $G \rightarrow 0$ & $0<G<G_{c r}$ & $G_{c r}<G<G^{*}$ & $G>G^{*}$ & $G \rightarrow \infty$ \\
\hline $\operatorname{Re}\left[\omega_{C S G}\right]$ & 0 & 0 & $>0, \omega_{C S G}<\omega_{L D G}$ & $>0, \omega_{C S G}>\omega_{L D G}$ & $\sqrt{R\left(G / G_{c r}-1\right)}$ \\
$\operatorname{Re}\left[\omega_{R T}\right]$ & 0 & $>0$ & $>0, \omega_{C S G}<\omega_{R T}$ & $>0, \omega_{C S G}>\omega_{R T}$ & $\omega_{R T}$ \\
$\operatorname{Re}\left[\omega_{L D G}\right]$ & $>0$ & $>0$ & $>0, \omega_{R T}<\omega_{L D G}$ & $>0, \omega_{R T}>\omega_{L D G}$ & $\omega_{R T}$ \\
\hline \hline
\end{tabular}

TABLE II. Properties of the accelerated conservative dynamics (CDG), accelerated Landau-Darrieus (LDG) dynamics, and Rayleigh-Taylor (RT) dynamics in the linear regime.

\begin{tabular}{|c|c|c|c|}
\hline & CDG & LDG & RT \\
\hline $\begin{array}{l}\text { Conservation } \\
\text { properties }\end{array}$ & $\begin{array}{l}\text { Conserves mass, momentum and energy at } \\
\text { the interface }\end{array}$ & $\begin{array}{l}\text { Conserves mass and momentum and has } \\
\text { zero perturbed mass flux at the interface }\end{array}$ & $\begin{array}{l}\text { Conserves mass and momentum and has } \\
\text { zero mass flux at the interface }\end{array}$ \\
\hline $\begin{array}{l}\text { Interface velocity of } \\
\text { the as a whole }\end{array}$ & Time-dependent & Constant & Zero \\
\hline Flow field & Potential velocity fields & Vortical structures in the light fluid & Potential velocity fields \\
\hline Interfacial shear & Shear-free & Shear-free & Interfacial shear \\
\hline Formal properties & $\begin{array}{l}\text { Non-degenerate; } 4 \text { fundamental solutions } \\
\text { and } 4 \text { degrees of freedom }\end{array}$ & $\begin{array}{l}\text { Degenerate; } 3 \text { fundamental solutions and } 4 \\
\text { degrees of freedom }\end{array}$ & $\begin{array}{l}\text { Degenerate; } 2 \text { fundamental solutions a } \\
\text { nd } 3 \text { degrees of freedom }\end{array}$ \\
\hline Stability & $\begin{array}{l}\text { Stability is set by the interplay of effects } \\
\text { of inertia (reactive force) and gravity. } \\
\text { Stable for small gravity values below } \\
\text { threshold; unstable for large gravity value } \\
\text { above threshold; largest growth-rate for } \\
\text { large gravity values }\end{array}$ & $\begin{array}{l}\text { Unstable for any gravity value, including } \\
\text { zero gravity value; largest growth-rate for } \\
\text { small gravity values; smallest growth-rate } \\
\text { for large gravity values }\end{array}$ & $\begin{array}{l}\text { Unstable for any gravity value, neutrally } \\
\text { stable for zero gravity }\end{array}$ \\
\hline
\end{tabular}

TABLE III. For the accelerated conservative dynamics with fixed values of $V_{h}, g, \sigma, \rho_{h}, \rho_{l}$, the values of the critical wavevector $k_{c r}$, at which the interface is stabilized, and the maximum wavevector $k_{\max }$ at which the unstable interface has the fastest growth. The ratio is $\left(k_{c r} / k_{\max }\right)=2$.

\begin{aligned} & \hline \hline Critical wavevector $k_{c r}=\left(\frac{g}{V_{h}^{2}}\right)\left(\frac{\rho_{l}}{\rho_{h}}\right)\left(\frac{\rho_{h}+\rho_{l}}{\rho_{h}-\rho_{l}}\right) \\ &$ Maximum wavevector $k_{\max }=\frac{1}{2}\left(\frac{g}{V_{h}^{2}}\right)\left(\frac{\rho_{l}}{\rho_{h}}\right)\left(\frac{\rho_{h}+\rho_{l}}{\rho_{h}-\rho_{l}}\right) \\ &$\hline\end{aligned}

a partial time-derivative), such that $\theta=0$ at the interface, and the heavy (light) fluid is located in the region $\theta>0$ $(\theta<0)$; its fields are $(\rho, \mathbf{v}, P, E) \rightarrow(\rho, \mathbf{v}, P, E)_{h(l)}$ and are marked with subscript $h(l)$ hereafter. ${ }^{4,11,24,31}$ By using the Heaviside step-function $H(\theta)$, we represent in the fluid fields in the entire domain as $(\rho, \mathbf{v}, P, E)=(\rho, \mathbf{v}, P, E)_{h} H(\theta)$ $+(\rho, \mathbf{v}, P, E){ }_{l} H(-\theta){ }^{24,31}$ At the interface, the balance of fluxes of mass and normal and tangential components of momentum and energy obey the boundary conditions $3,4,24,34$

$$
\begin{aligned}
{[\tilde{\mathbf{j}} \cdot \mathbf{n}]=0, } & {\left[\left(P+(\tilde{\mathbf{j}} \cdot \mathbf{n})^{2} / \rho\right) \mathbf{n}\right]=0, } \\
{[(\tilde{\mathbf{j}} \cdot \mathbf{n})((\tilde{\mathbf{j}} \cdot \tau) / \rho) \tau] } & =0, \quad\left[(\tilde{\mathbf{j}} \cdot \mathbf{n})\left(W+\tilde{\mathbf{j}}^{2} / 2 \rho^{2}\right)\right]=0,
\end{aligned}
$$

where $[\ldots]=0$ denotes the jump of functions across the interface; $\mathbf{n}$ and $\tau$ are the unit vectors normal and tangential at the interface with $\mathbf{n}=\nabla \theta /|\nabla \theta|$ and $(\mathbf{n} \cdot \boldsymbol{\tau})=0 ; \tilde{\mathbf{j}}$ $=\rho(\mathbf{n} \dot{\theta} /|\nabla \theta|+\mathbf{v})$ is the mass flux across the moving interface; $W=e+P / \rho$ is the specific enthalpy in its physics definition; particularly, the value $W$ includes the enthalpy of formation. 3,24

Here, we provide (the first, to our knowledge) systematic discussion of the boundary conditions Eq. (2a), including their direct derivation from the governing equations Eq. (1) and their important particular case of the zero mass flux at the interface, for revealing the full consistency of our approach with the classic results. ${ }^{3}$

In particular, we provide some details on how the boundary conditions Eq. (2a) are derived from the governing equations Eq. (1). We consider only the first of these conditions here; other conditions can be derived likewise. . $^{3,4,24,31,34}$ In particular, in the entire domain the fluid density is $\rho=\rho_{h} H(\theta)+\rho_{l} H(-\theta),{ }^{4,24,34}$ leading to

$$
\begin{aligned}
\partial \rho / \partial t= & \left(\partial \rho_{h} / \partial t\right) H(\theta)+\left(\partial \rho_{l} / \partial t\right) H(-\theta) \\
& +\delta(\theta)\left(\rho_{h}-\rho_{l}\right)(\partial \theta / \partial t)
\end{aligned}
$$

where it is accounted for that $\partial H(\theta) / \partial \theta=\delta(\theta)$ and $\delta(\theta)=\delta(-\theta)$, with $\delta(\theta)$ being the Dirac delta-function. By calculating other terms, substituting these expressions in the mass conservation equation in Eq. (1), and using $\partial \theta / \partial x_{i}=\nabla \theta=\mathbf{n}|\nabla \theta|$, we find

$$
\begin{aligned}
\partial \rho / \partial t+\partial \rho v_{i} / \partial x_{i}= & \left(\partial \rho / \partial t+\partial \rho v_{i} / \partial x_{i}\right)_{h} H(\theta) \\
& +\left(\partial \rho / \partial t+\partial \rho v_{i} / \partial x_{i}\right)_{l} H(-\theta) \\
& +\delta(\theta)|\nabla \theta|[\tilde{\mathbf{j}} \cdot \mathbf{n}]=0
\end{aligned}
$$

and reduce further to the conditions in the bulk of the heavy (light) fluid and at the interface 




FIG. 1. Schematics of the flow dynamics in a far field approximation (not to scale). Blue color marks the planar (dashed line) interface and the perturbed (solid line) interface.

$$
\begin{aligned}
& \left(\partial \rho / \partial t+\partial \rho v_{i} / \partial x_{i}\right)_{h}=0, \quad\left(\partial \rho / \partial t+\partial \rho v_{i} / \partial x_{i}\right)_{l}=0, \\
& {[\tilde{\mathbf{j}} \cdot \mathbf{n}]=0 .}
\end{aligned}
$$

The boundary conditions Eq. (2a) are derived from the governing equations Eq. (1) assuming that the mass flux is conserved at the interface, $[\tilde{\mathbf{j}} \cdot \mathbf{n}]=\left[\tilde{j}_{n}\right]=0$, with $\tilde{\mathbf{j}}=\rho(\mathbf{n} \dot{\theta} /$ $|\nabla \theta|+\mathbf{v})$ and $\tilde{j}_{n}=\tilde{\mathbf{j}} \cdot \mathbf{n}$. There is the important particular case, when the mass flux is conserved at the interface and when it is zero at the interface, $\left.\tilde{j}_{n}\right|_{\theta=0^{ \pm}}=0$. As is seen from Eq. (2a), this special condition leads to the continuity of the normal component of velocity at the interface $[\mathbf{v} \cdot \mathbf{n}]=0$ and transforms

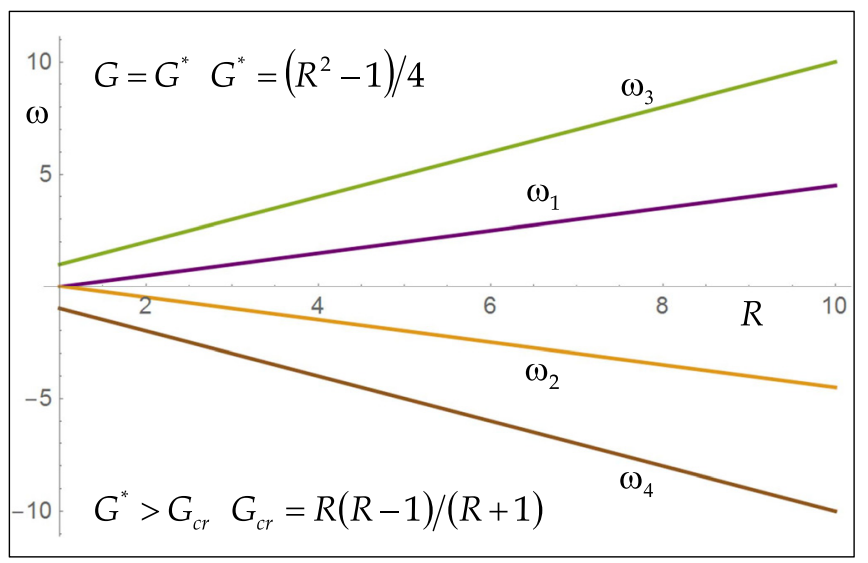

FIG. 2. Dependence of eigenvalues $\omega_{i}, i=1,2,3,4$, on the density ratio $R$ at gravity value $G=G^{*}, G^{*}=\left(R^{2}-1\right) / 4$, with $G^{*}>G_{c r}, G_{c r}=R(R$ $-1) /(R+1)$, for the conservative dynamics. the condition for the conservation of the normal component of momentum at the interface to the continuity of pressure at the interface, $[P]=0$. Moreover, for the zero mass flux at the interface, $\left.\tilde{j}_{n}\right|_{\theta=0^{ \pm}}=0$, the condition of continuity of the tangential component of momentum at the interface holds true for any jump of the tangential velocity at the interface $[\mathbf{v} \cdot \tau]=$ any, whereas the condition of continuity of the energy flux at the interface holds true for any jump of the enthalpy at the interface $[W]=$ any. Therefore, in full consistency with the classic results, ${ }^{3}$ in the case of zero mass flux at the interface, $\left.\tilde{j}_{n}\right|_{\theta=0^{ \pm}}=0$, the boundary conditions Eq. (2a) are transformed to

$$
[\tilde{\mathbf{j}} \cdot \mathbf{n}]=0, \quad[\mathbf{v} \cdot \mathbf{n}]=0, \quad[P \mathbf{n}]=0, \quad[\mathbf{v} \cdot \tau]=\text { any }, \quad[W]=\text { any } .
$$

The boundary conditions Eq. (2e) are applied to describe the dynamics a so-called contact discontinuity.

Our results Eqs. (2a) and (2e) are fully consistent with the classic results ${ }^{3}$ finding that there are two types of hydrodynamic discontinuities: (1) with the continuous mass flux, the continuous tangential velocity component and the discontinuous normal velocity component at the interface and (2) with the zero mass flux, the continuous normal velocity component and the discontinuous tangential velocity component at the interface. The classic results ${ }^{3}$ are derived in a frame of reference that moves with the velocity of the interface (see, e.g., Ref. 3 \#84); they are valid only at times when the interface velocity is constant. We derive the boundary conditions Eqs. (2a) and (2e) in an inertial frame of reference; they are valid at any time and for any interface velocity (and, given their covariant form, for any geometry).

In this work, we apply the conditions Eq. (2a) to study the general case of the dynamics of the interface with the interfacial mass flux in Secs. III A and III B, and the conditions Eq. (2e) to study the dynamics of the interface with zero interfacial mass flux in Sec. III C. Section III D compares the results.

We consider a spatially extended two-dimensional flow that propagates in the $z$ direction, is periodic in the $x$ direction, $x+\lambda \rightarrow x$, and has no motion in the $y$ direction, Fig. 1 . For the dynamics of the interface with the interfacial mass flux, in the inertial frame of reference, the boundary conditions at the outside boundaries of the domain are

$$
\left.\mathbf{v}_{h}\right|_{z \rightarrow-\infty}=\mathbf{V}_{h}=\left(0,0, V_{h}\right),\left.\quad \mathbf{v}_{l}\right|_{z \rightarrow+\infty}=\mathbf{V}_{l}=\left(0,0, V_{l}\right),
$$

with constant $V_{h(l)}$, Fig. 1. The initial conditions are the initial flow fields at the interface and in the bulk. They set the characteristic length-scale and time-scale of the dynamics. 3,20,23,24,34

Note that Eqs. (1)-(3) are derived in an inertial frame of reference that moves with a constant velocity $\tilde{\mathbf{V}}_{0}$. For the planar and steady interface, the velocity of this inertial frame of reference can be the interface velocity $\tilde{\mathbf{V}}$, as $\tilde{\mathbf{V}}_{0}=\tilde{\mathbf{V}}$. In general case of a non-steady non-planar interface, the interface velocity $\tilde{\mathbf{V}}$ and the velocity of the inertial frame of reference $\tilde{\mathbf{V}}_{0}$ are distinct values, $\tilde{\mathbf{V}} \neq \tilde{\mathbf{V}}_{0}$, and the interface velocity $\tilde{\mathbf{V}}$ obeys the relation $\tilde{\mathbf{V}} \mathbf{n}=-\left.\mathbf{v n}\right|_{\theta \rightarrow 0^{+}}=-\left.(\tilde{\mathbf{j}} / \rho) \mathbf{n}\right|_{\theta \rightarrow 0^{+}} .^{3,4,17,24,34}$ 
(a)

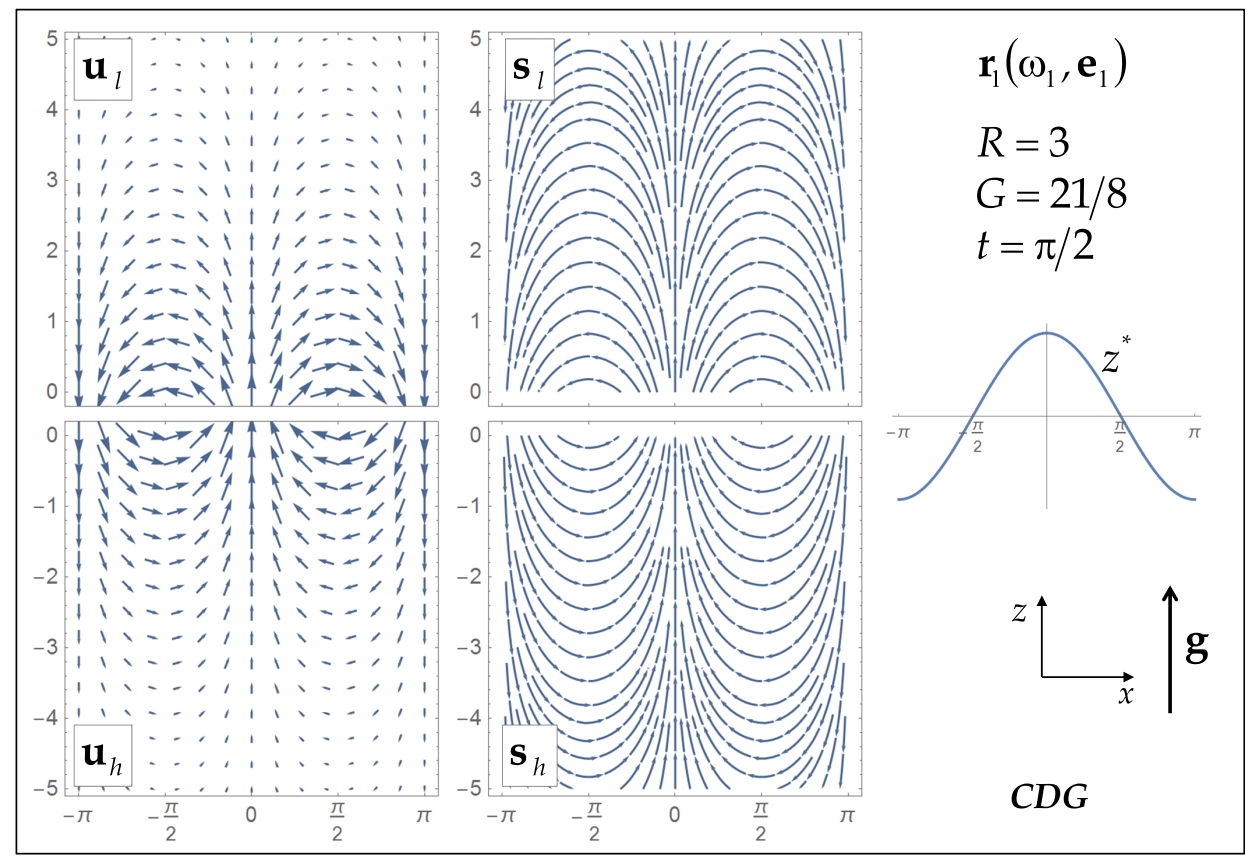

(b)

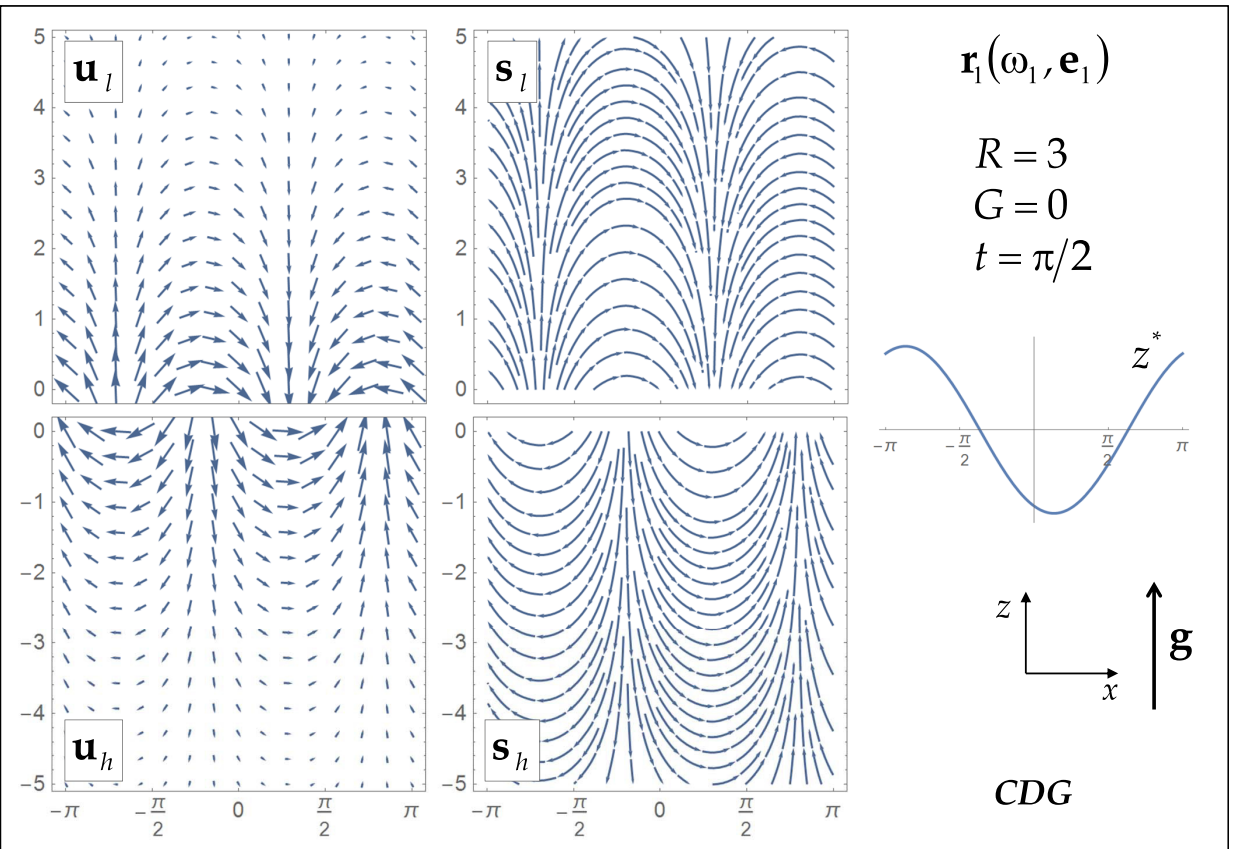

FIG. 3. Plots of the perturbed velocity vector fields $\mathbf{u}_{h(l)}$, the perturbed velocity streamlines $\mathbf{s}_{h(l)}$, and the interface perturbation $z^{*}$ in the $(x, z)$ plane for fundamental solution $\mathbf{r}_{1}$ for the accelerated conservative dynamics at some density ratio and some acceleration value at some instance of time. Real parts of fields and functions are shown. The velocity vortical component is $\nabla \times \Psi_{l}=0$, and the vorticity is $\nabla \times \mathbf{u}_{h(l)}=0$. (a) $i=1$, the unstable regime; (b) $i=1$, stable regime. Each plot has its own range of values to better present the plot's features.

There is also an important particular case, for which the velocity $\tilde{\mathbf{V}}_{0}$ is the same as the velocity of the steady planar interface, and for which the heavy fluid is at rest in the laboratory frame of reference. These assumptions lead to $\tilde{\mathbf{V}}_{0}=-\mathbf{V}_{h}$ with $\tilde{\mathbf{V}}_{0}=\left(0,0,-V_{h}\right) .^{3,4,17,24,34}$ In our general framework, the velocity $\tilde{\mathbf{V}}_{0}$ is understood as the velocity of the inertial frame of reference in the governing equations. ${ }^{24,34}$ This velocity can also be equal to the velocity of the unperturbed planar steady interface. Hereafter, for simplicity, we set the velocity of the inertial frame of reference equal to the velocity of the planar steady interface.

\section{B. Linearized dynamics}

We define $\theta=-z+z^{*}(x, t)$, with $\nabla \theta=\left(\partial z^{*} / \partial x, 0,-1\right)$, $|\nabla \theta|=\sqrt{1+\left(\partial z^{*} / \partial x\right)^{2}}, \dot{\theta}=\partial z^{*} / \partial t$. We slightly perturb the flow fields as $\tilde{\mathbf{j}}=\mathbf{J}+\mathbf{j}, \quad P=P_{0}+p, W=W_{0}+w$, with $|\mathbf{j}| \ll|\mathbf{J}|, \quad|p| \ll\left|P_{0}\right|, \quad|w| \ll\left|W_{0}\right|$, and $\mathbf{v}=\mathbf{V}+\mathbf{u}$ with $|\mathbf{u}| \ll|\mathbf{V}|$. We slightly perturb the interface as $|\dot{\theta} /| \nabla \theta|| \ll|\mathbf{V}|$ and $\left|\partial z^{*} / \partial x\right| \ll 1$, leading to $\mathbf{n}=\mathbf{n}_{0}+\mathbf{n}_{1}$ and $\tau=\tau_{0}+\tau_{1}$ with $\left|\mathbf{n}_{1}\right| \ll\left|\mathbf{n}_{0}\right|$ and $\left|\tau_{1}\right| \ll\left|\tau_{0}\right|$, Eqs. (1) and (2).

To the zeroth order, the mass flux is $\mathbf{J}=\rho \mathbf{V}$, and the flow fields are uniform in the bulk, $(\rho, \mathbf{v}, P, W)_{h(l)}$ 
(a)

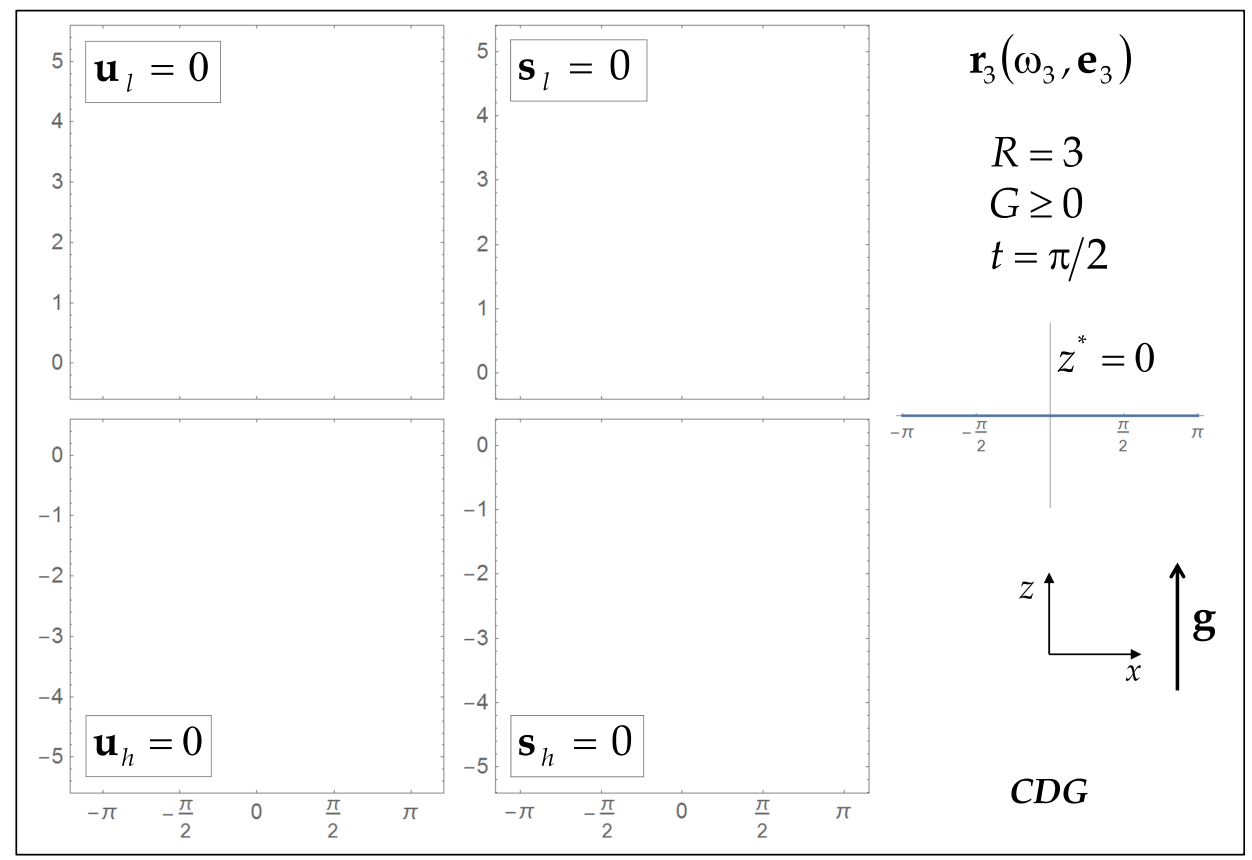

(b)



FIG. 4. Plots of the perturbed velocity vector fields $\mathbf{u}_{h(l)}$, the perturbed velocity streamlines $\mathbf{s}_{h(l)}$ and the interface perturbation $z^{*}$ in the $(x, z)$ plane for fundamental solutions $\mathbf{r}_{3(4)}$ for the accelerated conservative dynamics at some density ratio and some instance of time. Real parts of fields and functions are shown (a) $i=3$; (b) $i=4$. Each plot has its own range of values to better present the plot's features.

$=\left(\rho, \mathbf{V}, P_{0}, W_{0}\right)_{h(l)}$ and obey conditions Eq. (3) at the outside boundaries of the domain. The interface is planar, $\theta=-z, \quad \mathbf{n}=\mathbf{n}_{0} \quad$ and $\tau=\tau_{0}$ with $\mathbf{n}_{0}=(0,0,-1)$ and $\tau_{0}=(1,0,0)$. With $J_{n}=\mathbf{J} \cdot \mathbf{n}_{0}$, the boundary conditions at the interface are

$$
\begin{aligned}
& {\left[J_{n}\right]=0, \quad\left[\left(P_{0}+J_{n}^{2} / \rho\right) \mathbf{n}_{0}\right]=0,} \\
& {\left[J_{n}\left(\left(\mathbf{J} \cdot \tau_{0}\right) / \rho\right) \tau_{0}\right]=0, \quad\left[J_{n}\left(W_{0}+\mathbf{J}^{2} / 2 \rho^{2}\right)\right]=0 .}
\end{aligned}
$$

The boundary conditions Eqs. (2)-(4) are general and are applicable for both compressible and incompressible dynamics. ${ }^{3,24,34}$ In compressible flows, Eq. (4) are the Rankine-Hugoniot conditions.

In this work, we consider the incompressible fluids, in which the speed of sound is substantially greater than other velocity scales, and the effects of density variations and stratification are negligible. For ideal gases, the speed of sound is $c=\sqrt{\gamma P / \rho}$, where $\gamma$ is the fluid's adiabatic index. For the leading order dynamics, in the limiting incompressible case with $(c / V)_{h(l)} \rightarrow \infty$, the values approach $\left(P_{0}\right.$ $\left.+J_{n}^{2} / \rho\right)_{h(l)} \rightarrow\left(P_{0}\right)_{h(l)}$ and $\left(W_{0}+\mathbf{J}^{2} / 2 \rho^{2}\right)_{h(l)} \rightarrow\left(W_{0}\right)_{h(l)}$, and transform Eq. (4) to 
(a)

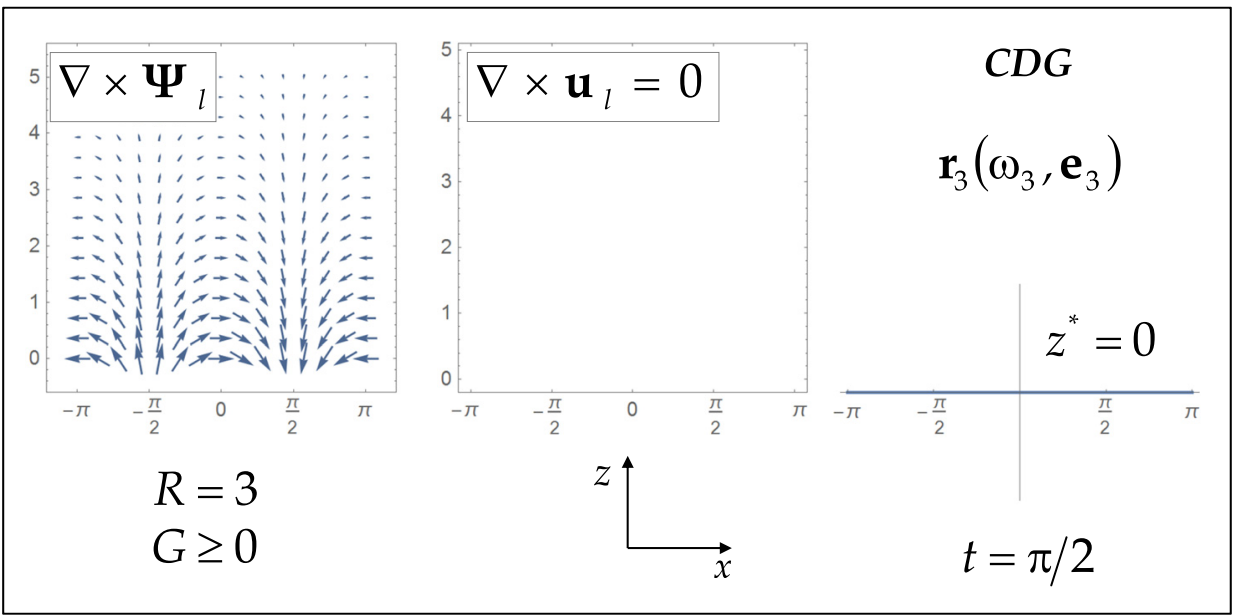

(b)



FIG. 5. Plots of the perturbed velocity vortical component $\nabla \times \boldsymbol{\Psi}_{l}$, the perturbed vorticity $\nabla \times \mathbf{u}_{l}$, and the interface perturbation $z^{*}$ in the $(x, z)$ plane for fundamental solutions $\mathbf{r}_{3(4)}$ for the accelerated conservative dynamics at some density ratio and some instance of time. Real parts of fields and functions are shown. While $\nabla \times \Psi_{l} \neq 0$, the vorticity value is $\nabla \times \mathbf{u}_{l}=0$. (a) $i=3$; (b) $i=4$. Each plot has its own range of values to better present the plot's features.

$$
\begin{aligned}
& {\left[J_{n}\right]=0, \quad\left[P_{0} \mathbf{n}_{0}\right]=0, \quad\left[J_{n}\left(\left(\mathbf{J} \cdot \tau_{0}\right) / \rho\right) \tau_{0}\right]=0,} \\
& {\left[J_{n} W_{0}\right]=0,}
\end{aligned}
$$

where the specific enthalpy is $\left(W_{0}\right)_{h(l)}=\left(\bar{W}_{0}+c_{P} T\right)_{h(l)}$. Here, $\left(c_{P}\right)_{h(l)}$ is the specific heat at constant pressure, $(T)_{h(l)}$ is temperature, and the values $\left(\bar{W}_{0}\right)_{h(l)}$ have a constant jump

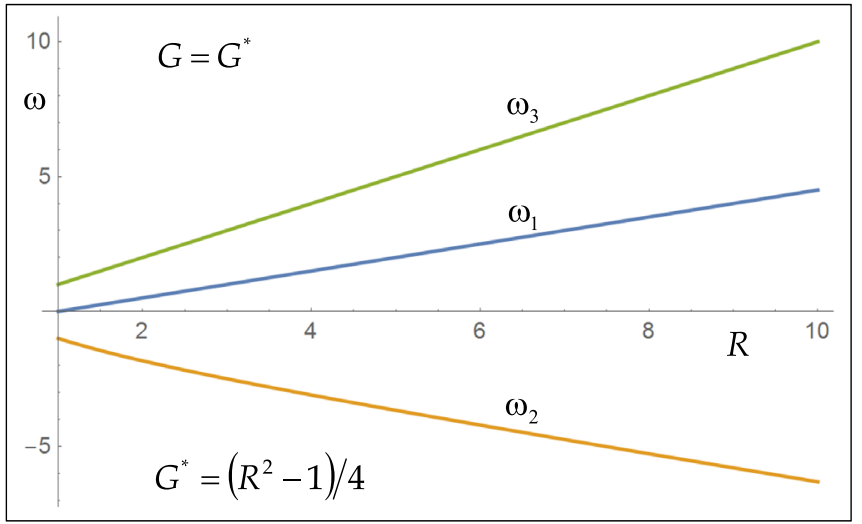

FIG. 6. Dependence of eigenvalues $\omega_{i}, i=1,2,3$, on the density ratio $R$ at gravity value $G=G^{*}, \quad G^{*}=\left(R^{2}-1\right) / 4$, with $G^{*}>G_{c r}, \quad G_{c r}=R(R$ $-1) /(R+1)$, for accelerated Landau-Darrieus dynamics. at the interface $\left[\bar{W}_{0}\right]=\bar{Q}$, where $\bar{Q}$ is the heat release per unit mass. In reactive fluids, the value $\bar{Q}$ is considered as the specific heat release of a chemical reaction at the absolute zero temperature. . $^{3,24,34}$

Note the difference between the definition of enthalpy $W_{0}$ in physics sense, which accounts for the formation enthalpy, and the enthalpy $\bar{W}_{0}$ applied in reactive fluids and engineering. The difference is discussed in detail in Ref. 3. In full consistency with this discussion, ${ }^{3}$ in the incompressible limit the physics enthalpy $W_{0}$ is continuous at the interface $\left[W_{0}\right]=0$, whereas the enthalpy $\bar{W}_{0}$ is discontinuous at the interface and its jump at the interface is $\left[\bar{W}_{0}\right]=\bar{Q}$.

To the first order, the boundary conditions at the interface are

$$
\begin{aligned}
& {\left[j_{n}\right]=0, \quad\left[\left(p+2 J_{n} j_{n} / \rho\right) \mathbf{n}_{0}\right]=0,} \\
& {\left[J_{n}\left(\mathbf{J} \cdot \tau_{1}+\mathbf{j} \cdot \tau_{0}\right) / \rho\right]=0, \quad\left[J_{n}\left(w+(\mathbf{J} \cdot \mathbf{j}) / \rho^{2}\right)\right]=0,}
\end{aligned}
$$

where the first order perturbation of the mass flux is $\mathbf{j}=\rho\left(\mathbf{u}+\mathbf{n}_{0} \dot{\theta}\right)$; its normal component is $j_{n}=\mathbf{j} \cdot \mathbf{n}_{0}$. The first order terms for the normal and tangential vectors of the interface are $\mathbf{n}_{1}=\left(\partial z^{*} / \partial x, 0,0\right)$ and $\tau_{1}=\left(0,0, \partial z^{*} / \partial x\right)$. 
(a)

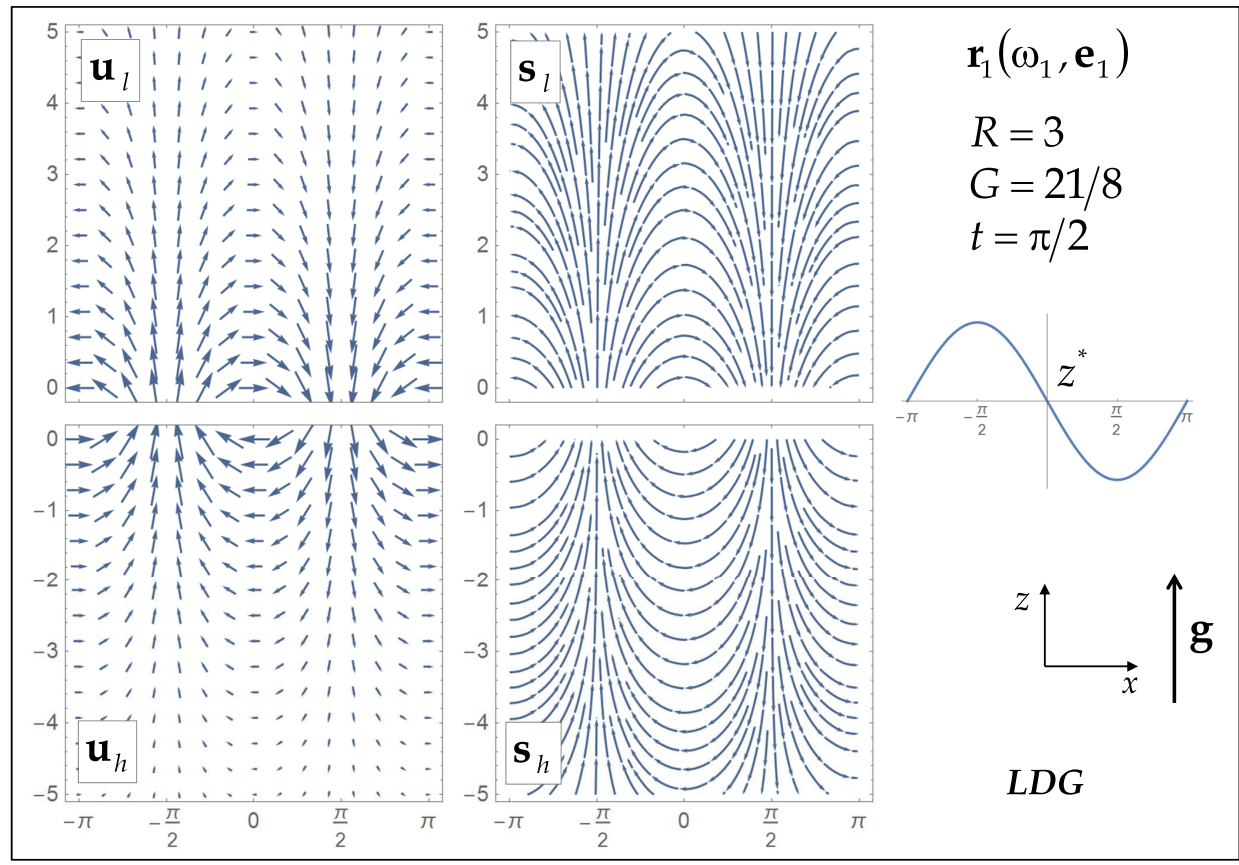

(b)



FIG. 7. Plots of the perturbed velocity vector fields $\mathbf{u}_{h(l)}$, the perturbed velocity streamlines $\mathbf{s}_{h(l)}$, and the interface perturbation $z^{*}$ in the $(x, z)$ plane for fundamental solutions $\mathbf{r}_{1(2)}$ for accelerated Landau-Darrieus dynamics at some density ratio and some acceleration value at some instance of time. Real parts of fields and functions are shown. (a) $i=1$; (b) $i=2$. Each plot has its own range of values to better present the plot's features.

In addition to the boundary conditions at the interface Eq. (6a), to the first order, upon presuming the ideal incompressible perturbed dynamics with neglected contributions of density variations and stratification, the governing equations in the bulk and at the outside boundaries take the form ${ }^{3,14,24}$

$$
\begin{array}{ll}
\nabla \cdot \mathbf{u}_{h(l)}=0, & \dot{\mathbf{u}}_{h(l)}+\left(\mathbf{V}_{h(l)} \cdot \nabla\right) \mathbf{u}_{h(l)}+\nabla p_{h(l)} / \rho_{h(l)}=0, \\
\left.\mathbf{u}_{h}\right|_{z \rightarrow-\infty}=0, & \left.\mathbf{u}_{l}\right|_{z \rightarrow+\infty}=0,
\end{array}
$$

whereas the enthalpy perturbations in Eq. (6a) are

$$
w_{h(l)}=p_{h(l)} / \rho_{h(l)} .
$$

The interface velocity $\tilde{\mathbf{V}}$ is $\tilde{\mathbf{V}}=\tilde{\mathbf{V}}_{0}+\tilde{\mathbf{v}}$ with the zeroth and the first order terms $\tilde{\mathbf{V}}_{0}$ and $\tilde{\mathbf{v}}$, with $|\tilde{\mathbf{v}}| \ll\left|\tilde{\mathbf{V}}_{0}\right|$, and with the first order term obeying the relations $\tilde{\mathbf{v}} \mathbf{n}_{0}=-\left.\left(\mathbf{u} \cdot \mathbf{n}_{0}+\dot{\theta}\right)\right|_{\theta=0^{+}}$.

\section{Fundamental solutions}

We seek solutions for the boundary value problem Eqs. (5) and (6) in which the perturbed velocity of the heavy fluid 
(a)

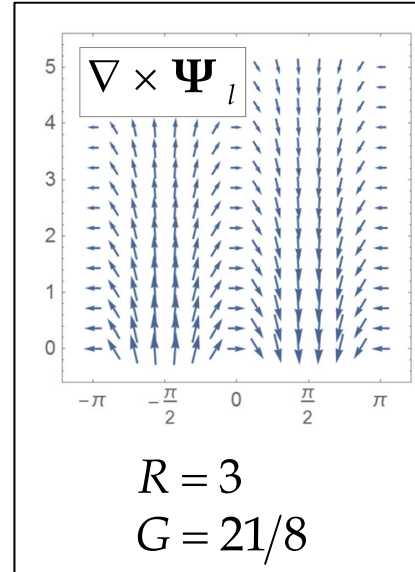

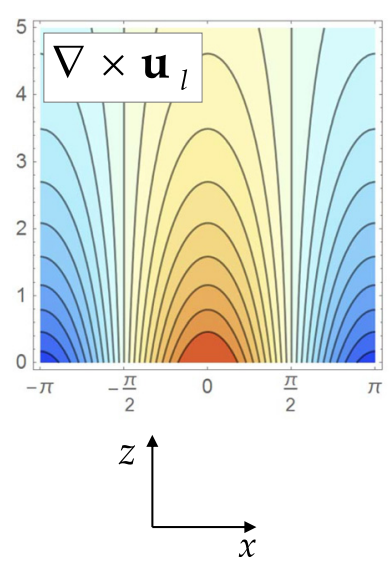

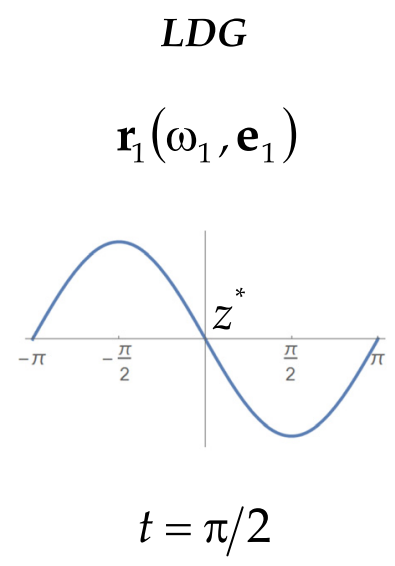

(b)



FIG. 8. Plots of the perturbed velocity vortical component $\nabla \times \boldsymbol{\Psi}_{l}$, the counterplot of the perturbed vorticity $\nabla \times \mathbf{u}_{l}$, and the interface perturbation $z^{*}$ in the $(x, z)$ plane for fundamental solutions $\mathbf{r}_{1(2)}$ for accelerated Landau-Darrieus dynamics at some density ratio and some acceleration value at some instance of time. Real parts of fields and functions are shown; in the counterplots the red (blue) color marks positive (negative) values. (a) $i=1$; (b) $i=2$. Each plot has its own range of values to represent the plots' features.

is potential, and the perturbed velocity of the light fluid has both potential and vortical components $3,17,20,23,24,34$

$$
\mathbf{u}_{h}=\nabla \Phi_{h}, \quad \mathbf{u}_{l}=\nabla \Phi_{l}+\nabla \times \boldsymbol{\Psi}_{l} .
$$

This structure of the solution Eq. (7) is based on the following rationale: In the sub-domain of the heavy fluid, the flow



FIG. 9. Dependence of eigenvalues $\omega_{i}, i=1,2$, on the density ratio $R$ at gravity value $G=G^{*}, G^{*}=\left(R^{2}-1\right) / 4$, for the Rayleigh-Taylor dynamics. is potential in accordance with the Kelvin theorem. 3,20,23,24,34 In the sub-domain of the light fluid, the flow can be a superposition of the potential and vortical components. The solution structure is applied in the theoretical framework, ${ }^{3,20}$ is consistent with observations, ${ }^{3,17,23,24}$ and is expected to be established for any initial conditions. ${ }^{17,23,24,34}$

The fluid potentials and the interface perturbation are

$$
\begin{aligned}
& \Phi_{h}=\Phi \exp (i k x+k z+\Omega t), \quad \Phi_{l}=\tilde{\Phi} \exp (i k x-k z+\Omega t), \\
& \boldsymbol{\Psi}_{l}=\left(0, \Psi_{l}, 0\right), \quad \Psi_{l}=\Psi \exp (i k x-\tilde{k} z+\Omega t), \\
& z^{*}=Z \exp (i k x+\Omega t) .
\end{aligned}
$$

Here, $\Omega$ is the growth-rate (the characteristic frequency, the eigenvalue) of the system of linear differential equations in Eq. (5); Fig. 1. Recall that the wavevector is $k=2 \pi / \lambda$ and the spatial period (wavelength) is $\lambda$. For the pressure $p_{h(l)}$ perturbations and the length-scale $\tilde{\lambda}=2 \pi / \tilde{k}$ of the vortical field, we obtain from Eq. (6)

$$
\begin{gathered}
\nabla\left(\dot{\Phi}_{h(l)}+V_{h(l)}\left(\partial \Phi_{h(l)} / \partial z\right)+p_{h(l)} / \rho_{h(l)}\right)=0, \\
\left(\partial / \partial t+V_{l}(\partial / \partial z)\right)\left(\nabla \times \Psi_{l}\right)=0,
\end{gathered}
$$




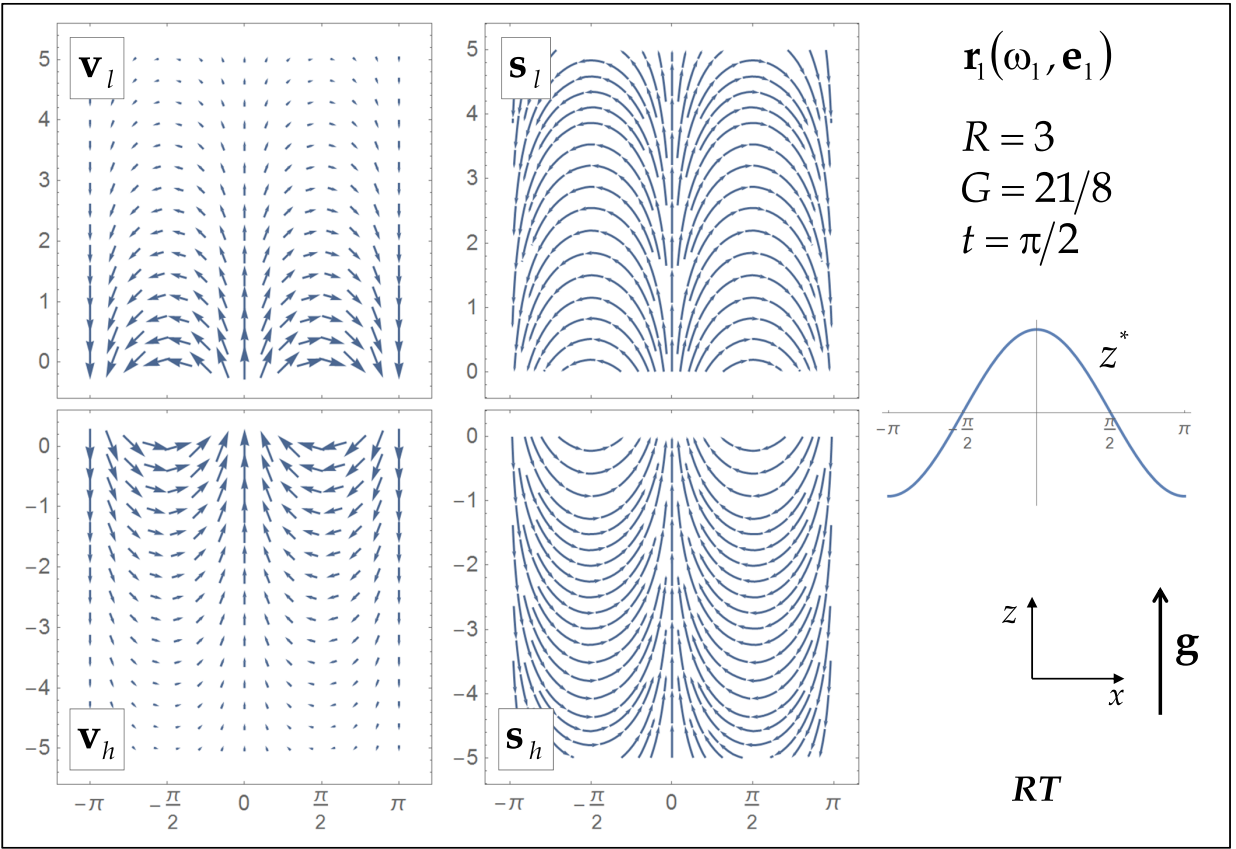

FIG. 10. Plots of the velocity vector fields $\mathbf{v}_{h(l)}$ and the velocity streamlines $\mathbf{s}_{h(l)}, \quad\left(d \mathbf{s}_{h(l)} / d t\right) \times \mathbf{v}_{h(l)}=0$, and the interface perturbation $z^{*}$ in $(x, z)$ plane at some density ratio and some acceleration value at some instance of time for the Rayleigh-Taylor dynamics. Real parts of fields and functions are shown. The vorticity is $\nabla \times \mathbf{v}_{h(l)}=0$ in the bulk, and the shear can be present at the interface. For solution $\mathbf{r}_{2}$, the flow fields are similar to that for solution $\mathbf{r}_{1}$. Each plot has its own range of values to better present the plot's features.

leading to

$$
p_{h(l)}=-\rho_{h(l)}\left(\dot{\Phi}_{h(l)}+V_{h(l)} \partial \Phi_{h(l)} / \partial z\right), \quad \tilde{k}=\Omega / V_{l}
$$

In the systems of equations Eqs. (1)-(7), the initial conditions set the characteristic length-scale $1 / k$ and time-scale $1 / k V_{h}$, Fig. 1. In the presence of acceleration $\mathbf{g}$ directed from the heavy to the light fluid, the pressure perturbations are modified as $p_{h(l)}=-\rho_{h(l)}\left(\dot{\Phi}_{h(l)}+V_{h(l)} \partial \Phi_{h(l)} / \partial z-g z\right)$. Hereafter, we use dimensionless values for the growth-rate $\omega=\Omega / k V_{h}$, the density ratio $R=\rho_{h} / \rho_{l}$ with $R \geq 1$, and the gravity value $G=g / k V_{h}^{2}$ with $G>0$. This leads to $V_{l} / V_{h}=R$ and $\tilde{k} / k=\omega / R^{3,17,20,23,24,34}$

Traditionally, the linear system of differential equations Eqs. (6) and (7) is solved by applying compatibility conditions: some eigenvalues $\omega_{i}$ are derived, and stability or instability of associated solutions is identified by using the criterion $\operatorname{Re}\left[\omega_{i}(R)\right]<0$ or $\operatorname{Re}\left[\omega_{i}(R)\right]>0 . .^{2,3,17,20-23} \mathrm{We}$ develop and apply the general matrix method to analyze the

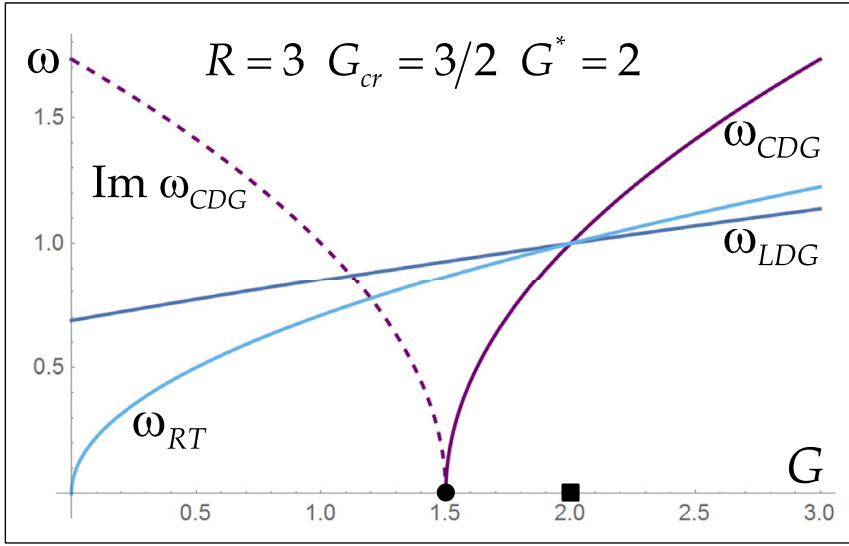

FIG. 11. Dependence of the growth-rates $\omega_{C S G}, \omega_{L D G}, \omega_{R T}$ on gravity value $G$. Density ratio $R=3$, square corresponds to $G^{*}=\left(R^{2}-1\right) / 4=3 / 2$, circle corresponds to $G_{c r}=R(R-1) /(R+1)=2$. interfacial dynamics, Eqs. (1)-(7). We find the fundamental solutions with their eigenvalues and eigenvectors and analyze the stability and the structure of the flow fields for each of these solutions. This formal analysis is required to better understand the interfacial dynamics. ${ }^{34}$

In particular, with expressions Eq. (7), the system of differential equations governing the interface dynamics Eqs. (5) and (6) is reduced to the linear system of ordinary differential equations $\mathbf{M r}=0$, where vector $\mathbf{r}$ is $\mathbf{r}=\left(\Phi_{h}\right.$, $\left.\Phi_{l}, V_{h} z^{*}, \Psi_{l}\right)^{\mathrm{T}}$, and the matrix $\mathrm{M}$ is defined by the boundary conditions at the interface. In the dimensionless form, its elements are the functions of the growth-rate (i.e., frequency, eigenvalue) $\omega$, the density ratio $R$, and the gravity value $G$, as $\mathbf{M}=\mathbf{M}(\omega, R, G) .{ }^{34}$ We derive the eigenvalues $\omega_{i}$ by using the condition det $\mathrm{M}\left(\omega_{i}, R, G\right)=0$, and further find the associated eigenvectors $\tilde{\mathbf{e}}_{i}$ by reducing the matrix $\mathrm{M}\left(\omega_{i}, R, G\right)$ to row-echelon form. ${ }^{34,35}$ Matrix $M$ is $4 \times 4$. For a nondegenerate $4 \times 4$ matrix its expected rank is 4 , and the expected number of eigenvalues $\omega_{i}$ and associated eigenvectors $\tilde{\mathbf{e}}_{i}$ is 4 , with $i=1 \ldots 4$, corresponding to 4 degrees of freedom and 4 independent variables obeying 4 equations, Eq. (6a).

Solution $\mathbf{r}$ for the system $\mathbf{M r}=0$ is a linear combination of fundamental solutions $\mathbf{r}_{i}$

$$
\mathbf{r}=\sum_{i=1}^{4} C_{i} \mathbf{r}_{i}
$$

with $\quad \mathbf{r}_{i}=\left(\Phi_{i} e^{i k x+k z+\omega_{i} k V_{h} t}, \tilde{\Phi}_{i} e^{i k x-k z+\omega_{i} k V_{h} t}, V_{h} Z_{i} e^{i k x+\omega_{i} k V_{h} t}\right.$ $\left.\Psi_{i} e^{i k x-\tilde{k} z+\omega_{i} k V_{h} t}\right)^{\mathrm{T}}$ in the dimensional units. In the dimensionless units, by introducing $\phi_{i}=\Phi_{i} /\left(V_{h} / k\right), \tilde{\phi}_{i}=\tilde{\Phi}_{i} /\left(V_{h} / k\right)$, $\bar{z}=k Z, \psi_{i}=\Psi_{i} /\left(V_{h} / k\right)$ and with $k x \rightarrow x, k z \rightarrow z$, $k V_{h} t \rightarrow t$, the solution is $\mathbf{r}_{i}=\left(\phi_{i} e^{i x+z+\omega_{i} t}, \tilde{\varphi}_{i} e^{i x-z+\omega_{i} t}\right.$, $\left.\bar{z}_{i} e^{i x+\omega_{i} t}, \psi_{i} e^{i x-(\tilde{k} / k) z+\omega_{i} t}\right)^{\mathrm{T}}$ with $C_{i}$ being integration constants, which are set by, e.g., the initial conditions. ${ }^{24,34}$ The fundamental solution is $\mathbf{r}_{i}=\tilde{\mathbf{e}}_{i} e^{\omega_{i} t}$ with $\tilde{\mathbf{e}}_{i}=\left(\phi_{i} e^{i x+z}\right.$, $\left.\tilde{\varphi}_{i} e^{i x-z}, \bar{z}_{i} e^{i x}, \psi_{i} e^{i x-(\tilde{k} / k) z}\right)^{\mathrm{T}}, \quad$ and $\quad \mathbf{r}_{i}=\mathbf{r}_{i}\left(\omega_{i}, \mathbf{e}_{i}\right)$ with $\mathbf{e}_{i}=\left(\phi_{i}, \tilde{\phi}_{i}, \bar{z}_{i}, \psi_{i}\right)^{\mathrm{T}}$. 


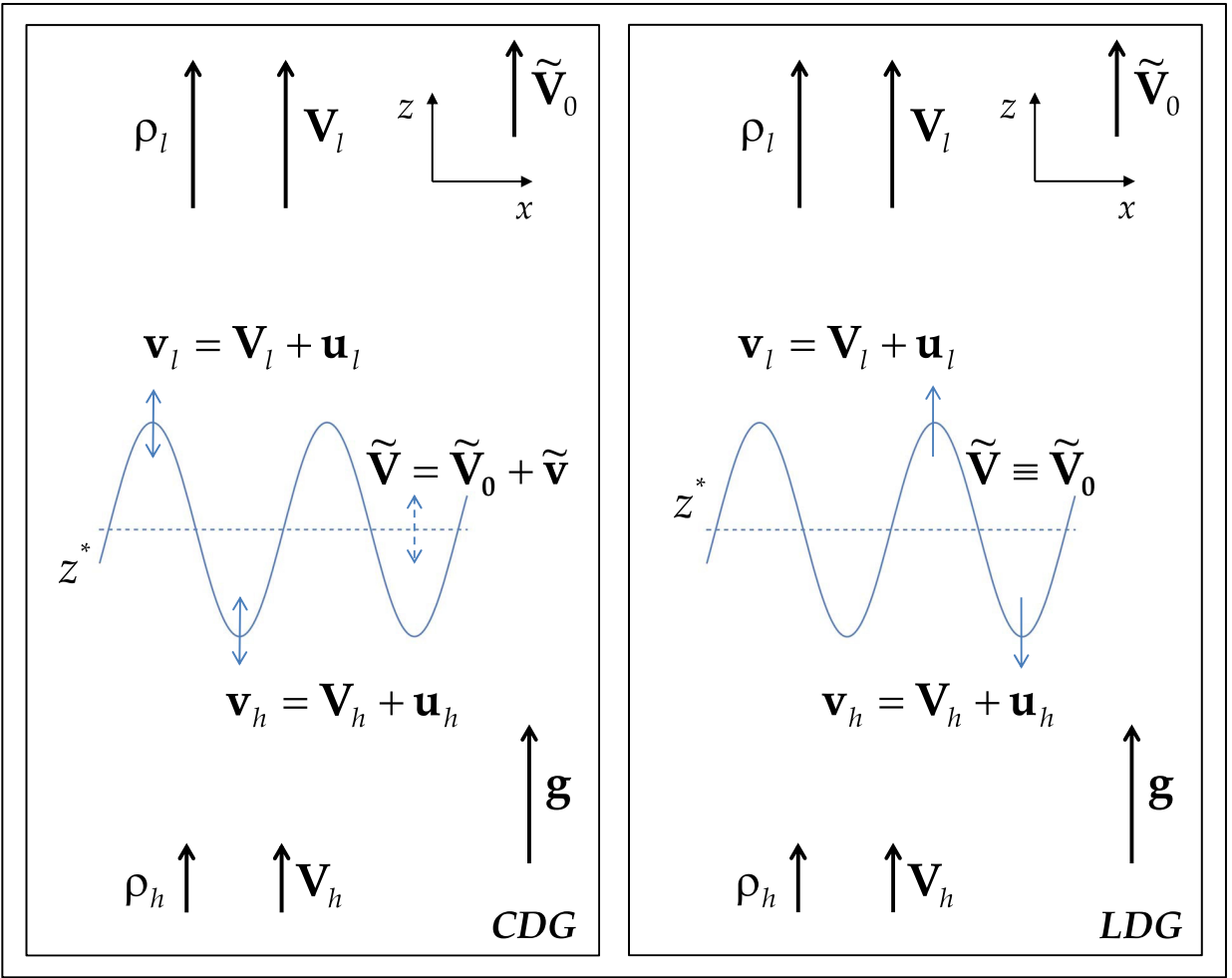

FIG. 12. Schematics of the flow dynamics in a far field approximation (not to scale) for the accelerated conservative dynamics (left) and accelerated Landau-Darrieus dynamics (right) in the inertial reference frame. Blue color marks the planar interface (dashed) and the perturbed interface (solid). For the conservative dynamics, the blue double arrows mark the oscillations of the interface perturbations (solid) and the interface velocity as a whole (dashed) with the latter occurring due to inertial effects and causing the reactive force to occur. For the accelerated Landau-Darrieus dynamics, the single blue arrows mark the growth of the interface perturbations the velocity of the interface as a whole is postulated constant.

Note that the linear system $M \mathbf{r}=0$ with $\mathbf{M}=\mathbf{M}(\omega$, $R, G)$ results from a linear system $\mathrm{P} \dot{\mathbf{r}}=\mathrm{Sr}$, where $\mathrm{P}, \mathrm{S}$ are $4 \times 4$ matrixes, with $\mathrm{P}=\mathrm{P}(R, G), \mathrm{S}=\mathrm{S}(R, G)$, under an assumption that vector $\mathbf{r}$ varies in time as $\mathbf{r} \sim e^{\omega t} .{ }^{24,34,35}$ This leads to $\mathrm{M}=(\mathrm{S}-\omega \mathrm{P})$. In a non-degenerate case, the inverse $\mathrm{P}^{-1}$ exists, and the system $\mathrm{P} \dot{\mathbf{r}}=\mathrm{Sr}$ can be reduced to a standard form $\dot{\mathbf{r}}=\mathrm{P}^{-1} \mathrm{Sr}$. The eigenvalues $\omega_{i}$ of the dynamics can then be found from the conditions $\operatorname{det}\left(\mathrm{P}^{-1} \mathrm{~S}\right.$ $-\omega \mathrm{I})=0, \omega=\left\{\omega_{i}\right\}$, where the unit matrix is I, and index $i$ marks the degrees of freedom. Equations $\operatorname{det}\left(\mathrm{P}^{-1} \mathrm{~S}-\omega \mathrm{I}\right)$ $=0$ and $\operatorname{det} \mathrm{M}(\omega, R)=0$ have the same eigenvalues $\omega_{i}$ because linear combination of differential equations preserves the dynamic properties. ${ }^{3,35}$

Our theoretical approach applies the general form of the expansions, Eqs. (1)-(8) in order to conduct a detailed and systematic study of the interplay of the interface stability with the structure of the flow fields, and to reveal the linkage of the stability of the interfacial dynamics to the flow vector and scalar fields at the interface and in the fluid bulk. ${ }^{34}$

\section{RESULTS}

\section{A. Accelerated conservative dynamics}

First, we consider the conservative dynamics balancing the fluxes of mass, momentum, and energy at the interface, Eq. (6a). For this dynamics, the matrix $\mathrm{M}$ has the form $\mathrm{M}=M_{G}$

$$
M_{G}=\left(\begin{array}{cccc}
-R & -1 & -\omega+R \omega & i \\
1 & -1 & 1-R & i \omega / R \\
R-R \omega & R+\omega & G(R-1) & -2 i R \\
\omega & -\omega & \omega-R \omega & i R
\end{array}\right) .
$$

Its determinant is $\operatorname{det} M_{G}=i\left((R-1)^{2} / R\right)(\omega-R)(\omega+R)$ $\left(\omega^{2}+R\left(1-G / G_{c r}\right)\right)$, and $\omega_{i}$ and $\mathbf{e}_{i}$ are

$$
\begin{aligned}
\omega_{1(2)} & = \pm i \sqrt{R} \sqrt{1-G / G_{c r}}, \quad \mathbf{e}_{1(2)}=(*, *, 1,0)^{\mathrm{T}} ; \\
\omega_{3} & =R, \quad \mathbf{e}_{3}=(0, *, 0,1)^{\mathrm{T}} ; \quad \omega_{4}=-R, \quad \mathbf{e}_{4}=(*, *, 0,1)^{\mathrm{T}},
\end{aligned}
$$

where $G_{c r}=R(R-1) /(R+1)$ and asterisks mark functions of $R, G$. Figure 2 illustrates the dependence of eigenvalues $\omega_{i}$ on the density ratio $R$ in Eq. (8) for $G=G^{*}$, $G^{*}=\left(R^{2}-1\right) / 4, G^{*}>G_{c r}$.

For the accelerated conservative dynamics Eq. (8), fundamental solutions $\mathbf{r}_{1}\left(\omega_{1}, \mathbf{e}_{1}\right)$ and $\mathbf{r}_{2}\left(\omega_{2}, \mathbf{e}_{2}\right)$ depend on the value $G$. For $G<G_{c r}$, the eigenvalues are imaginary, and the solutions $\mathbf{r}_{1(2)}$ are stable, with $\omega_{2}=\omega_{1}^{*}$ and $\mathbf{e}_{2}=\mathbf{e}_{2}^{*}$. For $G>G_{c r}, \omega_{1}=\sqrt{R} \sqrt{G / G_{c r}-1}$ and $\operatorname{Re}\left[\omega_{1}\right]>0$, so the solution $\mathbf{r}_{1}$ is unstable and describes exponential growth of the interface perturbations, whereas $\omega_{2}=-\sqrt{R} \sqrt{G / G_{c r}-1}$ and $\operatorname{Re}\left[\omega_{2}\right]<0$, so the solution $\mathbf{r}_{2}$ is stable and describes exponential decay of the perturbations. The critical value $G_{c r}=R(R-1) /(R+1)$ approaches $G_{c r} \rightarrow R$ for $R \rightarrow \infty$ and approaches $G_{c r} \rightarrow(R-1) / 2$ for $R \rightarrow 1^{+}$. Fundamental solutions $\mathbf{r}_{3}\left(\omega_{3}, \mathbf{e}_{3}\right)$ and $\mathbf{r}_{4}\left(\omega_{4}, \mathbf{e}_{4}\right)$ in Eq. (8) do not depend on the value $G$. Solution $\mathbf{r}_{3}$ is unstable, $\omega_{3}=R$ and $\operatorname{Re}\left[\omega_{3}\right]>0$, whereas solution $\mathbf{r}_{4}$ is stable, $\omega_{4}=-R$ and $\operatorname{Re}\left[\omega_{4}\right]<0$.

Figures 3-5 illustrate the flow fields of fundamental solutions $\mathbf{r}_{i}\left(\omega_{i}, \mathbf{e}_{i}\right)$ for the accelerated conservative dynamics in Eq. (8). Figure 3 presented for fundamental solutions $\mathbf{r}_{1}$ in the unstable and stable regimes the plots of the perturbed velocity vector fields $\mathbf{u}_{h(l)}$ and the perturbed velocity streamlines $\mathbf{s}_{h(l)}$, which are defined as $\left(d \mathbf{s}_{h(l)} / d t\right) \times \mathbf{u}_{h(l)}=0$, and the interface perturbation $z^{*}$ in the $(x, z)$ plane at some density ratio and some acceleration value at some instance of time. Real parts of fields and functions are shown. For solution $\mathbf{r}_{1}$, the velocity fields are potential in the heavy and the 
light fluids, and the vorticity $\nabla \times \mathbf{u}_{h(l)}=0$. For solution $\mathbf{r}_{2}\left(\omega_{2}, \mathbf{e}_{2}\right)$, the velocity fields are qualitatively similar to those for $\mathbf{r}_{1}\left(\omega_{1}, \mathbf{e}_{1}\right)$. For these solutions, the transports of mass, momentum, and energy are balanced at the interface, and the conditions at the outside boundaries are obeyed, Eqs. (1)-(7). Note that in the unstable regime, $G>G_{c r}$, the solutions $\mathbf{r}_{1(2)}$ are standing waves with the growing (decaying) amplitude(s), Fig. 3(a). In the stable regime, $G<G_{c r}$, the solutions $\mathbf{r}_{1(2)}$ are traveling waves, and their interference results in the appearance of standing waves experiencing stable oscillations, Fig. 3(b). Note also the phase shifts of the perturbed velocity fields relative one another and relative the interface perturbation for solutions $\mathbf{r}_{1(2)}$, Fig. 3(b). These phase shifts are required to balance the perturbed mass flux across the perturbed interface in the incompressible approximation, Fig. 3.

Figure 4 presents for fundamental solutions $\mathbf{r}_{3(4)}$ the plots of the perturbed velocity vector fields $\mathbf{u}_{h(l)}$ and the perturbed velocity streamlines $\mathbf{s}_{h(l)}$, with $\left(d \mathbf{s}_{h(l)} / d t\right) \times \mathbf{u}_{h(l)}=0$, and the interface perturbation $z^{*}$ in the $(x, z)$ plane at some density ratio and some acceleration value at some instance of time. The solutions $\mathbf{r}_{3(4)}$ are independent of the acceleration value. For solution $\mathbf{r}_{3}\left(\omega_{3}, \mathbf{e}_{3}\right)$ in Eq. (8), the fluids' velocities and the interface perturbation are identically zero $\left(\mathbf{u}_{h(l)}=0, z^{*}=0\right)$ in the entire domain at any time for any integration constant $C_{3}$, Fig. 4(a). For solution $\mathbf{r}_{4}\left(\omega_{4}, \mathbf{e}_{4}\right)$ in Eq. (8), we must set the integration constant $C_{4}=0$, in order for this solution to obey at any time the conditions $\left.\mathbf{u}_{l}\right|_{z \rightarrow+\infty}=0$ in Eq. (6a); Fig. 4(b). This is because the velocity vortical component, $\nabla \times \boldsymbol{\Psi}_{l}$, while decaying in time, increases away from the interface, Fig. 4(b). Figure 5 further illustrates the properties of the vortical field $\boldsymbol{\Psi}_{l}$ for fundamental solutions $\mathbf{r}_{3(4)}$. Note that while the vortical component is $\nabla \times \boldsymbol{\Psi}_{l} \neq 0$, the vorticity value is $\nabla \times \mathbf{u}_{l}=0$, because $(\tilde{k} / k)^{2}=(\omega / R)^{2}=1$.

To study formal properties of the accelerated conservative dynamics, we find for matrix $\mathrm{M}=M_{G}$ the associated matrices $\mathrm{S}=S_{M_{G}}$ and $\mathrm{P}=P_{M_{G}}$

$$
\begin{aligned}
S_{M_{G}} & =\left(\begin{array}{cccc}
-R & -1 & 0 & i \\
1 & -1 & 1-R & 0 \\
R & R & G(R-1) & -2 i R \\
0 & 0 & 0 & i R
\end{array}\right), \\
P_{M_{G}} & =\left(\begin{array}{cccc}
0 & 0 & 1-R & 0 \\
0 & 0 & 0 & -i / R \\
R & -1 & 0 & 0 \\
-1 & 1 & -1+R & 0
\end{array}\right) .
\end{aligned}
$$

Solutions of equations $\operatorname{det}\left(\mathrm{P}_{M_{G}}^{-1} \mathrm{~S}_{M_{G}}-\omega \mathrm{I}\right)=0$ and $\operatorname{det} M_{G}$ $=0$ yield the same eigenvalues: $\omega_{1(2)}= \pm i \sqrt{R} \sqrt{1-G / G_{c r}}$ and $\omega_{3}=R, \omega_{4}=-R$. The accelerated conservative dynamics has 4 eigenvalues, 4 fundamental solutions, and 4 independent degrees of freedom, and is thus non-degenerate.

\section{B. Accelerated Landau-Darrieus instability}

For classical accelerated Landau-Darrieus instability, the dynamics balances the fluxes of mass, and normal and tangential components of momentum, and employs the special condition of the continuity of the normal component of the perturbed velocity $\left[\mathbf{u} \cdot \mathbf{n}_{0}\right]=0$ at the interface. . $^{3,20,23,24}$ This condition is equivalent to the constancy of the interface velocity, $\tilde{\mathbf{V}} \equiv \tilde{\mathbf{V}}_{0}{ }^{3,20,23,24}$ The Landau's dynamics omits from consideration the energy balance at the interface. The classical Landau's dynamics leads to

$$
\begin{aligned}
& {\left[j_{n}\right]=0, \quad\left[\left(p+2 J_{n} j_{n} / \rho\right) \mathbf{n}_{0}\right]=\left[p \mathbf{n}_{0}\right]=0,} \\
& {\left[J_{n}\left(\mathbf{J} \cdot \tau_{1}+\mathbf{j} \cdot \tau_{0}\right) / \rho\right]=0, \quad\left[\mathbf{u} \cdot \mathbf{n}_{0}\right]=0 .}
\end{aligned}
$$

With these conditions, for the classical Landau's system the matrix $\mathrm{M}$ is $\mathrm{M}=L_{G}$.

$$
L_{G}=\left(\begin{array}{cccc}
-R & -1 & -\omega+R \omega & i \\
1 & -1 & 1-R & i \omega / R \\
R-R \omega & R+\omega & G(R-1) & -2 i R \\
-1 & -1 & 0 & i
\end{array}\right) .
$$

Its determinant is $\operatorname{det} L_{G}=i((R-1) / R)(\omega-R)\left((R+1) \omega^{2}\right.$ $+2 R \omega-(R-1)(R+G))$, and $\omega_{i}$ and $\mathbf{e}_{i}$ are

$$
\begin{aligned}
& \omega_{1(2)}=\left(-R \pm \sqrt{\left(R^{3}+R^{2}-R\right)+G\left(R^{2}-1\right)}\right) /(1+R), \\
& \mathbf{e}_{1(2)}=(*, *, *, 1)^{\mathrm{T}} ; \quad \omega_{3}=R, \quad \mathbf{e}_{3}=(0, *, 0,1)^{\mathrm{T}},
\end{aligned}
$$

where asterisks mark functions of $R$ and $G$. In Eq. (9), the solutions $\mathbf{r}_{1(2)}\left(\omega_{1(2)}, \mathbf{e}_{1(2)}\right)$ depend on $G$, and the solution $\mathbf{r}_{3}\left(\omega_{3}, \mathbf{e}_{3}\right)$ is independent of $G$ and is identical to that in Eq. (8). Figure 6 illustrates the dependence of eigenvalues $\omega_{i}$ on the density ratio $R$ in Eq. (9) at $G=G^{*}, G^{*}=\left(R^{2}-1\right) / 4$, $G^{*}>G_{c r}$.

For the classical Landau's dynamics Eq. (9), fundamental solution $\mathbf{r}_{1}\left(\omega_{1}, \mathbf{e}_{1}\right)$ corresponds to accelerated LandauDarrieus instability in a gravity field and is unstable for any $G>0 .{ }^{3,20}$ For solution $\mathbf{r}_{1}$, the transports of mass, momentum, and perturbed mass flux are balanced at the interface and the conditions at the outside boundaries are obeyed, Eqs. (6a) and (9). The potential and vortical components of the velocities and the interface perturbation are strongly coupled; the length-scale of the vortical field is $\tilde{k}$ $=k\left(-R+\sqrt{\left(R^{3}+R^{2}-R\right)+G\left(R^{2}-1\right)}\right) /(R(1+R))$. For solution $\mathbf{r}_{2}\left(\omega_{2}, \mathbf{e}_{2}\right)$, the interface perturbation and the vortical and potential components of velocities are also coupled. For this solution, we must set the constant $C_{2}=0$ in order to obey at any time the condition $\left.\mathbf{u}_{l}\right|_{z \rightarrow+\infty}=0$ in Eq. (6a). Solution $\mathbf{r}_{3}\left(\omega_{3}, \mathbf{e}_{3}\right)$ in Eq. (9) is identical to that in Eq. (8) and has zero velocity fields in the entire domain for any integration constant $C_{3}$ and at any time.

Figures 7-8 illustrate the flow fields of fundamental solutions $\mathbf{r}_{1(2)}\left(\omega_{1(2)}, \mathbf{e}_{1(2)}\right)$ for the accelerated Landau-Darrieus dynamics in Eq. (9). The flow fields for solution $\mathbf{r}_{3}\left(\omega_{3}, \mathbf{e}_{3}\right)$ in Eq. (9) are identical to that in Eq. (8) and Figs. 3-5. Figure 7 presents for fundamental solutions $\mathbf{r}_{1(2)}$ the plots of the perturbed velocity vector fields $\mathbf{u}_{h(l)}$, the perturbed velocity streamlines $\mathbf{s}_{h(l)},\left(d \mathbf{s}_{h(l)} / d t\right) \times \mathbf{u}_{h(l)}=0$, and the interface perturbation $z^{*}$ in the $(x, z)$ plane at some density ratio and some acceleration value at some instance of time. Real parts 
of fields and functions are shown. For solutions $\mathbf{r}_{1}\left(\omega_{1}, \mathbf{e}_{1}\right)$ and $\mathbf{r}_{2}\left(\omega_{2}, \mathbf{e}_{2}\right)$, the velocity fields have the potential component in the heavy fluid and the potential and vortical components in the light fluid that are coupled with the interface perturbations.

Figure 8 further illustrates the properties of the vortical fields $\boldsymbol{\Psi}_{l}$ for fundamental solutions $\mathbf{r}_{1(2)}$ in Eq. (9). Note that for solutions $\mathbf{r}_{1(2)}$ the vorticity value is $\nabla \times \mathbf{u}_{l}=\left(0,\left(k^{2}\right.\right.$ $\left.\left.-\tilde{k}^{2}\right) \Psi, 0\right)$ and $\nabla \times \mathbf{u}_{l} \neq 0$. For solution $\mathbf{r}_{1}$, the vortical component, $\nabla \times \boldsymbol{\Psi}_{l}$, and the vorticity $\nabla \times \mathbf{u}_{l}$, while increasing in time as $\sim e^{\omega_{1} t}$, decay away from the interface as $\sim e^{-\tilde{k z}}$. For solution $\mathbf{r}_{2}$ the vortical component, $\nabla \times \boldsymbol{\Psi}_{l}$, while decaying in time as $\sim e^{-\left|\omega_{2}\right| t}$, increases away from the interface as $\sim e^{+\tilde{k} z}$. This leads to zero integration constant $C_{2}=0$ in order to obey at any time the boundary conditions at the outside boundaries of the domain, Eq. (6b).

The accelerated Landau-Darrieus dynamics has smaller than the expected number of fundamental solutions. To study formal properties of the dynamics, we find for matrix $\mathrm{M}=L_{G}$ the associated matrices $\mathrm{S}=S_{L_{G}}$ and $\mathrm{P}=P_{L_{G}}$

$$
\begin{aligned}
S_{L_{G}} & =\left(\begin{array}{cccc}
-R & -1 & 0 & i \\
1 & -1 & 1-R & 0 \\
R & R & G(R-1) & -2 i R \\
-1 & -1 & 0 & i
\end{array}\right), \\
P_{L_{G}} & =\left(\begin{array}{cccc}
0 & 0 & 1-R & 0 \\
0 & 0 & 0 & -i / R \\
R & -1 & 0 & 0 \\
0 & 0 & 0 & 0
\end{array}\right) .
\end{aligned}
$$

In matrix $P_{L_{G}}$, the fourth row only has null elements and $\operatorname{det} P_{L_{G}}=0$. Thus, the inverse matrix $P_{L_{G}}^{-1}$ does not exist. This suggests the degeneracy of the classical Landau's dynamics, and a singular and ill-posed character of the accelerated Landau-Darrieus instability. We address detailed consideration of this issue to future research.

\section{Rayleigh-Taylor instability}

For the classical Rayleigh-Taylor instability, the discontinuity is the front (contact discontinuity), and the dynamics has zero mass flux at the interface, as discussed in Eq. (2e). It also has zero motion of the fluids away from the interface..$^{3,4,25,26}$ This leads to

$$
\begin{aligned}
& {\left[\tilde{j}_{n}\right]=0, \quad[P \mathbf{n}]=0, \quad[\mathbf{v} \cdot \mathbf{n}]=0,[\mathbf{v} \cdot \tau]=\text { any },} \\
& {[W]=\text { any },\left.\quad \mathbf{v}_{h}\right|_{z \rightarrow-\infty}=(0,0,0),\left.\quad \mathbf{v}_{l}\right|_{z \rightarrow+\infty}=(0,0,0) .}
\end{aligned}
$$

The unperturbed velocity field is zero in both fluids. We slightly perturb the velocities with the potential fields, $\quad \mathbf{v}_{h}=\nabla \Phi_{h}, \quad \Phi_{h}=\Phi e^{i k x+k z+\Omega t}, \quad$ and $\quad \mathbf{v}_{l}=\nabla \Phi_{l}$, $\Phi_{l}=\tilde{\Phi} e^{i k x-k z+\Omega t}$, with $|\mathbf{v}| \ll \sqrt{g / k}$, and perturb the fluid pressure, $P=P_{0}+p,|p| \ll\left|P_{0}\right|$, with $p_{h(l)}=-\rho_{h(l)}\left(\dot{\Phi}_{h(l)}\right.$ $\left.+V_{h(l)} \partial \Phi_{h(l)} / \partial z-g z\right)$. We slightly perturb the interface $\theta=-z+z^{*}(x, t)$, with $z^{*}=Z e^{i k x+\Omega t}, \quad|\dot{\theta} /| \nabla \theta|| \ll \sqrt{g / k}$ and $\left|\partial z^{*} / \partial x\right| \ll 1$. Then system Eq. (10a) is reduced to a linear system $\mathbf{M r}=0$, where vector $\mathbf{r}$ is $\mathbf{r}=\left(\Phi_{h}, \Phi_{l}, V_{h} z^{*}\right)^{\mathrm{T}}$ and $3 \times 3$ matrix is $\mathrm{M}=\mathrm{M}(\omega, R, G)$. The solution is $\mathbf{r}=\sum_{i} C_{i} \mathbf{r}_{i}$, where $\mathbf{r}_{i}=\mathbf{r}_{i}\left(\omega_{i}, \mathbf{e}_{i}\right)$ are the fundamental solutions, $C_{i}$ are the integration constants, and $i=1,2,3$ in the non-degenerate case, similarly to Eq. (7e). For the RT dynamics in Eq. (10a), matrix $\mathrm{M}$ is $\mathrm{M}=T_{G}$

$$
T_{G}=\left(\begin{array}{ccc}
-R & -1 & -\omega+R \omega \\
-R-R \omega & -R+\omega & G(R-1) \\
-1 & -1 & 0
\end{array}\right) .
$$

Its determinant is $\operatorname{det} T_{G}=(R-1)\left((R+1) \omega^{2}-G(R-1)\right)$, and $\omega_{i}$ and $\mathbf{e}_{i}$ are

$$
\omega_{1(2)}= \pm \sqrt{G(R-1) /(R+1)}, \quad \mathbf{e}_{1(2)}=(*, *, 1)^{\mathrm{T}},
$$

where asterisks mark functions of $R, G$. Note that in RTI the length-scale and the time-scale are $1 / k$ and $1 / \sqrt{g k}$. In order to conduct a comparative study of the RT dynamics with the accelerated conservative dynamics and accelerated LandauDarrieus dynamics, we scale the time with $1 / k V_{h}$, where $V_{h}$ is now understood as some velocity scale. This leads to $G=g / k V_{h}^{2}$, as before. For any $G>0$, the solution $\mathbf{r}_{1}\left(\omega_{1}, \mathbf{e}_{1}\right)$ is unstable, whereas solution $\mathbf{r}_{2}\left(\omega_{2}, \mathbf{e}_{2}\right)$ is stable. Figure 9 illustrates the dependence of eigenvalues $\omega_{i}$ on the density ratio $R$ in Eq. (10) at $G=G^{*}, G^{*}=\left(R^{2}-1\right) / 4, G^{*}>G_{c r}$.

Figure 10 illustrates the flow fields of fundamental solution $\mathbf{r}_{1}\left(\omega_{1}, \mathbf{e}_{1}\right)$ for the Rayleigh-Taylor dynamics in Eq. (10) representing the plots of the velocity vector fields $\mathbf{v}_{h(l)}$ and the velocity streamlines $\mathbf{s}_{h(l)}$, with $\left(d \mathbf{s}_{h(l)} / d t\right) \times \mathbf{v}_{h(l)}=0$, and the interface perturbation $z^{*}$ in the $(x, z)$ plane at some density ratio and some acceleration value at some instance of time. Real parts of fields and functions are shown. For solution $\mathbf{r}_{1}$, the velocity fields are potential, $\nabla \times \mathbf{v}_{h(l)}=0$, and shear is present at the interface. For solution $\mathbf{r}_{2}$, the flow fields are similar to those for solution $\mathbf{r}_{1}$.

The Rayleigh-Taylor dynamics has smaller than the expected number of fundamental solutions. To study formal properties of the dynamics, we find for matrix $\mathrm{M}=T_{G}$ the associated matrices $\mathrm{S}=S_{T_{G}}$ and $\mathrm{P}=P_{T_{G}}$

$$
\begin{aligned}
S_{T_{G}} & =\left(\begin{array}{ccc}
-R & -1 & 0 \\
-R & -R & G(R-1) \\
-1 & -1 & 0
\end{array}\right), \\
P_{T_{G}} & =\left(\begin{array}{ccc}
0 & 0 & 1-R \\
R & -1 & 0 \\
0 & 0 & 0
\end{array}\right) .
\end{aligned}
$$

In matrix $P_{T_{G}}$, the third row only has null elements, determinant $\operatorname{det} P_{L_{G}}=0$, and the inverse matrix $P_{T_{G}}^{-1}$ does not exist. This suggests the degeneracy of RT dynamics, and a singular and ill-posed character of the RTI. We address detailed consideration of this issue to future research.

\section{Comparative study}

In each of the systems Eqs. (8)-(10), the solution $\mathbf{r}_{1}\left(\omega_{1}, \mathbf{e}_{1}\right)$ leads to the unstable dynamics. Yet, the 
conservative solution $\mathbf{r}_{1}\left(\omega_{1}, \mathbf{e}_{1}\right)$ in Eq. (8), hereafter $\mathbf{r}_{C S G}\left(\omega_{C S G}, \mathbf{e}_{C S G}\right)$, the Landau-Darrieus solution $\mathbf{r}_{1}\left(\omega_{1}, \mathbf{e}_{1}\right)$ in Eq. (9), hereafter $\mathbf{r}_{L D G}\left(\omega_{L D G}, \mathbf{e}_{L D G}\right)$, and the Rayleigh-Taylor solution $\mathbf{r}_{1}\left(\omega_{1}, \mathbf{e}_{1}\right)$ in Eq. (10), hereafter $\mathbf{r}_{R T}\left(\omega_{R T}, \mathbf{e}_{R T}\right)$, have distinct quantitative, qualitative, and formal properties.

\section{Quantitative properties}

The growth-rate value $\omega_{C S G}=\sqrt{G(R+1) /(R-1)-R}$ indicates that the stability of the dynamics $\mathbf{r}_{C S G}$ is defined by the interplay of the two terms, $\sim G$ and $\sim R$, i.e., the gravity and the inertia. In the $(G, R)$ plane, the stability curve for the solution is $f=0$, with $f=G-R(R-1) /(R+1)$, and for $G>0$ and $R>1$ the region of the (un)stable dynamics $\mathbf{r}_{C S G}$ is $f<0(f>0)$. At a given $G(R)$, there always exists the interval of values of $R$ $(G)$, where the accelerated conservative dynamics is unstable. The dynamics $\mathbf{r}_{L D G}$ with the growth-rate $\omega_{L D G}=(-R$ $\left.+\sqrt{\left(R^{3}+R^{2}-R\right)+G\left(R^{2}-1\right)}\right) /(1+R)$ and the dynamics $\mathbf{r}_{R T}$ with the growth-rate $\omega_{R T}=\sqrt{G(R-1) /(R+1)}$ contains no interplay of the gravity and inertia and are unstable for $G>$ 0 and $R>1$.

We consider the dependence of the dynamics $\mathbf{r}_{C S G}, \mathbf{r}_{R T}$, and $\mathbf{r}_{L D G}$ on the acceleration value $G, G \in(0,+\infty)$, for a given finite density ratio $R, R \in\left(1^{+},+\infty\right)$. For solution $\mathbf{r}_{C S G}$, the instability develops only when the acceleration value is greater than a threshold, $G>G_{c r}$, whereas for solutions $\mathbf{r}_{L D G}$ and $\mathbf{r}_{R T}$, the dynamics is unstable for any $G>0$. For $G \rightarrow 0$, the dynamics $\mathbf{r}_{C S G}$ is stable, $\omega_{C S G} \rightarrow i \sqrt{R}$, the dynamics $\quad \mathbf{r}_{L D G}$ is unstable $\omega_{L D G} \rightarrow(-R$ $\left.+\sqrt{R^{3}+R^{2}-R}\right) /(1+R)$; the dynamics $\mathbf{r}_{R T}$ is neutrally stable, $\omega_{R T} \rightarrow 0$. For $G \rightarrow \infty$, the dynamics $\mathbf{r}_{C S G}, \mathbf{r}_{R T}$, and $\mathbf{r}_{L D G}$ are unstable; the growth-rates are $\omega_{C S G} \rightarrow \sqrt{R G / G_{c r}}$, $\omega_{R T}=\sqrt{G(R-1) /(R+1)}$, and $\omega_{L D G} \rightarrow \omega_{R T}$. There exists a special value of $G=G^{*}$ at which all the growth-rates are equal, $\omega_{C S G}=\omega_{L D G}=\omega_{R T}$. This value is $G^{*}=\left(R^{2}-1\right) / 4$, $G^{*}>G_{c r}$. For $G_{c r}<G<G^{*}$, solution $\mathbf{r}_{C S G}$ has the smallest growth-rate, $\omega_{C S G}<\omega_{R T}<\omega_{L D G}$. For $G>G^{*}$, solution $\mathbf{r}_{C S G}$ has the largest growth-rate, $\omega_{C S G}>\omega_{R T}>\omega_{L D G}$, Fig. 11. For $G \rightarrow \infty$, the unstable conservative dynamics has the fastest growth-rate and $\omega_{R T(L D G)} / \omega_{C S G} \rightarrow(R-1) /(R+1)$, Fig. 11. These properties are summarized in Table I.

We consider the properties for the dynamics $\mathbf{r}_{C S G}, \mathbf{r}_{R T}$, and $\mathbf{r}_{L D G}$ in the limiting cases of fluids with very similar and very different densities. For $R \rightarrow 1^{+}$, the dynamics $\mathbf{r}_{C S G}$ is (un)stable for $G<G_{c r}\left(G>G_{c r}\right)$, with $G_{c r} \rightarrow(R-1) / 2$, and the dynamics $\mathbf{r}_{L D G}$ and $\mathbf{r}_{R T}$ are unstable, with $\omega_{L D G} \rightarrow$ $(1+G)(R-1) / 2$ and $\omega_{R T} \rightarrow \sqrt{G(R-1) / 2}$. At $R \equiv 1$, the dynamics $\mathbf{r}_{C S G}$ is neutrally stable conditional $G / G_{C r}<1$ for $R \rightarrow 1^{+}$with $G_{C r}=(R-1) / 2$; the dynamics $\mathbf{r}_{L D G}$ is neutrally stable for $G \geq 0$; the dynamics $\mathbf{r}_{R T}$ is neutrally stable conditional there is zero initial shear at the interface. For $R \rightarrow \infty$, the dynamics $\mathbf{r}_{C S G}$ is (un)stable for $G<G_{c r}$ $\left(G>G_{c r}\right)$ with $G_{c r} \rightarrow R$; the dynamics $\mathbf{r}_{L D G}$ and $\mathbf{r}_{R T}$ are unstable, with $\omega_{L D G} \rightarrow \sqrt{R}$ and $\omega_{R T} \rightarrow \sqrt{G}$, Fig. 11; Table I.

\section{Qualitative properties}

Solutions $\mathbf{r}_{C S G}, \mathbf{r}_{L D G}$, and $\mathbf{r}_{R T}$ have distinct flow fields in the bulk and at the interface. In the bulk, solutions $\mathbf{r}_{C S G}$ and $\mathbf{r}_{R T}$ have potential velocity fields, whereas for solution $\mathbf{r}_{L D G}$ the potential and vortical components of the velocity fields are strongly coupled. At the interface, solutions $\mathbf{r}_{C S G}$ and $\mathbf{r}_{L D G}$ are shear-free, whereas solution $\mathbf{r}_{R T}$ has the interfacial shear leading to the development of Kelvin-Helmholtz instability at the interface (and the roll-up of vortices in the nonlinear regime). ${ }^{4,11,29-31}$ These properties are discussed in the foregoing Eqs. (8)-(10), and are illustrated by Figs. 3-5, 7, 8 , and 10 and summarized in Table II.

\section{Interfacial shear}

To better understand the properties of the interfacial shear for the accelerated conservative dynamics $\mathbf{r}_{C S G}$ and accelerated Landau-Darrieus dynamics $\mathbf{r}_{L D G}$, we consider in more detail the condition of continuity of the tangential component of momentum in Eq. (2a) for the interface with the interfacial mass flux, as well as the zeroth and the first order terms of the expansions of this condition in Eqs. (5), (6a), and (9a).

In Eq. (2a), since $[\tilde{\mathbf{j}} \cdot \mathbf{n}]=0$ and $\tilde{\mathbf{j}}=\rho(\mathbf{n} \dot{\theta} /|\nabla \theta|+\mathbf{v})$, the condition of continuity of the tangential component of momentum at the interface $[(\tilde{\mathbf{j}} \cdot \mathbf{n})((\tilde{\mathbf{j}} \cdot \tau) / \rho) \tau]=0$ is equivalent to the condition of continuity of the tangential component of velocity at the interface $[\mathbf{v} \cdot \tau]=0$, in full consistency with the classic results. ${ }^{3}$ To the zeroth order in Eq. (5), the condition $\left[J_{n}\left(\left(\mathbf{J} \cdot \tau_{0}\right) / \rho\right) \tau_{0}\right]=0$ is transformed to $\left[\mathbf{V} \cdot \tau_{0}\right]=0$; it is identically zero due to $\mathbf{V} \cdot \tau_{0}=V\left(\mathbf{n}_{0} \cdot \tau_{0}\right)$ $=0$. To the first order in Eqs. (6a) and (9a), the condition $\left[J_{n}\left(\mathbf{J} \cdot \tau_{1}+\mathbf{j} \cdot \tau_{0}\right) / \rho\right]=0$ is $\left[J_{n}\left(\mathbf{V} \cdot \tau_{1}+\mathbf{u} \cdot \tau_{0}\right)\right]=0$. Since $\left[J_{n}\right]=0$, it is reduced to the condition $\left[\mathbf{V} \cdot \tau_{1}+\mathbf{u} \cdot \tau_{0}\right]=0$. The latter is the first order component of the tangential velocity $\mathbf{v}=\mathbf{V}+\mathbf{u}$ at the perturbed interface with the tangential vector $\tau=\tau_{0}+\tau_{1}$, where $\tau_{0}=(1,0,0)$ and $\tau_{1}=\left(0,0, \partial z^{*} / \partial x\right)$.

We see that while the tangential component of the perturbed velocity $\mathbf{u} \cdot \tau_{0}$ may have a jump at the interface, the tangential component of the velocity $\mathbf{v}$ is continuous at the interface $\left[\mathbf{V} \cdot \tau_{1}+\mathbf{u} \cdot \tau_{0}\right]=0$, and the dynamics is shear-free at the interface for both the accelerated conservative dynamics and accelerated Landau-Darrieus dynamics, Eqs. (6a) and (9a); Figs. 3(a), 3(b), and 7(a).

To better understand the properties of the interfacial shear for the Rayleigh-Taylor dynamics $\mathbf{r}_{R T}$, we consider in some detail the important particular case of the zero mass at the interface in Eq. (2e) and (10a). As discussed in Sec. II A and in Eq. (2e), the condition of the zero mass flux leads to the discontinuity of the tangential velocity component and the discontinuity of enthalpy at the interface, $[\mathbf{v} \cdot \tau]=$ any and $[W]=$ any. This case corresponds to the RayleighTaylor dynamics in Eq. (10a), for which the tangential component of velocity has a jump at the interface, $[\mathbf{v} \cdot \tau]=$ any, and leads to the interfacial shear for solution $\mathbf{r}_{R T}$, Eq. (10) and Fig. 10.

These properties of the interfacial shear are clearly illustrated by Figs. 3(a), 7(a), and 10 representing solutions $\mathbf{r}_{C S G}$, 
$\mathbf{r}_{L D G}$ and $\mathbf{r}_{R T}$. Figures 3(a) and 7(a) [and, similarly, Fig. 3(b) for the stable traveling wave $\mathbf{r}_{1}$ ] illustrate for solutions $\mathbf{r}_{C S G}$ and $\mathbf{r}_{L D G}$ the field of the perturbed velocity $\mathbf{u}$, whose tangential component $\mathbf{u} \cdot \tau_{0}$ may have a jump at the interface, while keeping continuous the tangential component of the fluid velocity $\mathbf{v}$, since $\left[\mathbf{V} \cdot \tau_{1}+\mathbf{u} \cdot \tau_{0}\right]=0$, and the shear-free interfacial dynamics, as prescribed by the boundary conditions Eqs. (2a), (6a), and (9a). Figure 10 illustrates for solution $\mathbf{r}_{R T}$ the field of the fluid velocity $\mathbf{v}$, for which the tangential component of the velocity has a jump at the interface $[\mathbf{v} \cdot \tau]=$ any, and leads to the interfacial shear, as prescribed by the boundary conditions Eq. (10a).

We emphasize that while the accelerated conservative dynamics and accelerated Landau-Darrieus dynamics are shear-free at the interface, for the accelerated conservative dynamics $\mathbf{r}_{C S G}$ the velocity field is potential, Fig. 3(a), whereas accelerated Landau-Darrieus dynamics $\mathbf{r}_{L D G}$ has the vortical field $\boldsymbol{\Psi}_{l} \neq 0$ and the vorticity $\nabla \times \mathbf{v}_{l}=\nabla \times \mathbf{u}_{l} \neq 0$ in the light fluid bulk, Figs. 7(a) and 8(a). For accelerated Landau-Darrieus dynamics $\mathbf{r}_{L D G}$ the vorticity field achieves extreme values at the interface and decays away from the interface, Fig. 8(a). In Rayleigh-Taylor dynamics $\mathbf{r}_{R T}$ the velocity fields are potential in the fluids' bulk, the vortical structures are interfacial in nature and develop only at the interface due to the shear caused by the discontinuity of the tangential component of velocity at the interface, Fig. 10.

\section{Formal properties}

Solutions $\mathbf{r}_{C S G}, \mathbf{r}_{L D G}$, and $\mathbf{r}_{R T}$ have distinct formal properties, as discussed in the foregoing. Physically, solution $\mathbf{r}_{C S G}$ conserves mass, momentum, and energy at the interface; solution $\mathbf{r}_{L D G}$ conserves mass, momentum, and perturbed mass flux at the interface; solution $\mathbf{r}_{R T}$ conserves mass and normal component of momentum at the interface, Eqs. (8)-(10). Mathematically, the conservative dynamics is non-degenerate (4 fundamental solutions, 4 degrees of freedom); the LD dynamics is degenerate (3 solutions, 4 degrees of freedom); the RT dynamics is degenerate ( 2 solutions, 3 degrees of freedom), Eqs. (8)-(10). These properties are discussed in detail in the foregoing and are summarized in Table II.

\section{E. Mechanisms of stabilization and destabilization}

To understand the mechanisms of stabilization and destabilization of the accelerated conservative dynamics, we transfer to dimensional units and consider solution $\mathbf{r}_{C S G}$ at zero acceleration value, $g=0$. In this case, when the interface is slightly perturbed, the parcels of the heavy and the light fluids follow the interface perturbation, thus causing the change of momentum of the fluid system. Yet, at zero acceleration value the dynamics is inertial and the momentum should be conserved. To conserve the momentum, the interface as a whole slightly changes its velocity, thus causing a reactive force to occur. ${ }^{34}$ For the acceleration value $g>0$ the interface stability is set by the interplay of inertia and gravity. For $g<g_{c r}$ the inertia effects dominate, and the dynamics is stable. For $g>g_{c r}$, the gravity effects dominate, and the dynamics is unstable. The inertial stabilization mechanism is absent in the accelerated LDI and the classical RTI, because in these cases in the linear regime the velocity of the interface as a whole is a constant value, and in RTI this value is zero. Figure 12 illustrates the mechanism of stabilization and destabilization. ${ }^{34}$

For the accelerated conservative dynamics, the interface stability is defined by the interplay of the effect of inertia and gravity (i.e., the reactive force and gravity), and the growth-rate (the frequency) of the dynamics is $\Omega_{C D G}=k V_{h} \sqrt{\left(\rho_{h} / \rho_{l}\right)\left(g / g_{c r}-1\right)}$. For small gravity values, $g / g_{c r}<1$, the inertial effects dominate, and the dynamics is stable, $\Omega_{C D G}=i k V_{h} \sqrt{\left(\rho_{h} / \rho_{l}\right)\left(1-g / g_{c r}\right)}$. For large gravity values, $g / g_{c r}>1$, the gravity effects dominate and the dynamics is unstable $\Omega_{C D G}=k V_{h} \sqrt{\left(\rho_{h} / \rho_{l}\right)\left(g / g_{c r}-1\right)}$. The critical gravity value is $g_{c r}=k V_{h}^{2}\left(\rho_{h} / \rho_{l}\right)\left(\rho_{h}-\rho_{l}\right) /\left(\rho_{h}+\rho_{l}\right)$.

For given values of $V_{h}, g, \rho_{h}, \rho_{l}$, the inertial stabilization mechanism establishes the values of the critical wavevector $k_{c r}$ at which the interface is stabilized and the maximum wavevector $k_{\max }$ at which the unstable interface has the fastest growth. We find the critical wavevector $k_{c r}$ from the condition $\left.\Omega_{C D G}\right|_{k=k_{c r}}=0$. We find the maximum wavevector $k_{\max }$ from the conditions $\partial \Omega_{C D G} /\left.\partial k\right|_{k=k_{\max }}=0, \partial^{2} \Omega_{C D G} /$ $\left.\partial k^{2}\right|_{k=k_{\max }}<0$. Their values are

$$
\begin{aligned}
k_{c r} & =\left(g / V_{h}^{2}\right)\left(\rho_{l} / \rho_{h}\right)\left(\rho_{h}+\rho_{l}\right) /\left(\rho_{h}-\rho_{l}\right), \\
k_{\max } & =\left(g / V_{h}^{2}\right)\left(\rho_{l} / \rho_{h}\right)\left(\rho_{h}+\rho_{l}\right) / 2\left(\rho_{h}-\rho_{l}\right), \\
k_{\max } & =k_{c r} / 2 .
\end{aligned}
$$

Thus, for the accelerated conservative dynamics in ideal incompressible fluids in the absence of traditional stabilization mechanisms (including, e.g., surface tension, diffusion, thermal transport, viscosity) the values of the critical wavevector and the maximum wavevector are finite, and their ratio is $k_{c r} / k_{\max }=2$, Table III. For accelerated LandauDarrieus and Rayleigh-Taylor dynamics in ideal incompressible fluids the inertial stabilization mechanism is absent, and, without traditional stabilization mechanisms, the values of the critical wavevector and the maximum wavevector are infinitely large, and $k_{c r(\max )} /\left(g / V_{h}^{2}\right) \rightarrow \infty$.

To conclude this Section, for the purposes of completeness, we provide the solutions $\mathbf{r}_{C D G(L D G, R T)}$ with $\omega_{C D G(L D G, R T)}$ and $\mathbf{e}_{C D G(L D G, R T)}=\left(\phi_{i}, \tilde{\phi}_{i}, \bar{z}_{i}, \psi_{i}\right)_{C D G(L D G, R T)}^{\mathrm{T}}$ in Table IV.

\section{DISCUSSION}

In this work, we have developed and applied the general matrix method for analyzing the stability of a hydrodynamic discontinuity. We have considered the stability of the accelerated interface that separates the incompressible ideal fluids of different densities, and has the interfacial mass flux, Eqs. (1)-(11); Tables I-IV; Figs. 1-12. The acceleration (gravity) is directed the heavy to the light fluid. The case of a twodimensional spatially period flow is studied. The far field approximation is applied. The effects of density variations and stratification are assumed to be negligible. We have developed the general theoretical framework to rigorously solve the system of the governing equations that include the 
TABLE IV. Solutions $\mathbf{r}_{C D G(L D G, R T)}$ with $\omega_{C D G(L D G, R T)}$ and $\mathbf{e}_{C D G(L D G, R T)}=\left(\varphi_{i}, \tilde{\varphi}_{i}, \bar{z}_{i}, \psi_{i}\right)_{C D G(L D G, R T)}^{\mathrm{T}}$.

Accelerated conservative dynamics $\mathbf{r}_{C D G}\left(G>G_{c r}\right)$

$$
\mathbf{e}_{C D G}=\left(\begin{array}{c}
\omega_{C D G}=\sqrt{\frac{-R(R-1)+G(R+1)}{R-1}} \\
\frac{-R(R-1)+\sqrt{(R-1)(-R(R-1)+G(R+1))}}{R+1} \\
R+1 \\
1
\end{array}\right)
$$

Accelerated Landau-Darrieus dynamics $\mathbf{r}_{L D G}$

$$
\mathbf{e}_{L D G}=\left(\begin{array}{c}
\omega_{L D G}=\frac{-R+\sqrt{\left(R^{3}+R^{2}-R\right)+G\left(R^{2}-1\right)}}{1+R} \\
i \frac{\left(R-\sqrt{R\left(R^{2}+R-1\right)+G\left(R^{2}-1\right)}\right)\left(\left(R^{2}+2 R\right)-\sqrt{R\left(R^{2}+R-1\right)+G\left(R^{2}-1\right)}\right)}{R(R+1)\left(\left(R^{2}+2 R-1\right)-2 \sqrt{R\left(R^{2}+R-1\right)+G\left(R^{2}-1\right)}\right)} \\
-i \frac{(R-1)\left(R\left(\sqrt{R\left(R^{2}+R-1\right)+G\left(R^{2}-1\right)}-\left(R^{2}+2 R\right)\right)+G(R+1)\right)}{R(R+1)\left(\left(R^{2}+2 R-1\right)-2 \sqrt{R\left(R^{2}+R-1\right)+G\left(R^{2}-1\right)}\right)} \\
-i \frac{\left(\left(R^{2}+2 R\right)-\sqrt{R\left(R^{2}+R-1\right)+G\left(R^{2}-1\right)}\right)}{R\left(\left(R^{2}+2 R-1\right)-2 \sqrt{R\left(R^{2}+R-1\right)+G\left(R^{2}-1\right)}\right)} \\
1
\end{array}\right)
$$

Rayleigh-Taylor dynamics $\mathbf{r}_{R T}$

$$
\omega_{R T}=\sqrt{G \frac{R-1}{R+1}} \quad \mathbf{e}_{R T}=\left(\begin{array}{c}
\sqrt{G \frac{R-1}{R+1}} \\
-\sqrt{G \frac{R-1}{R+1}} \\
1
\end{array}\right)
$$

conservation of mass, momentum, and energy in the fluids' bulk and at the interface. We focus on the linear dynamics, with the slight perturbations of the interface and the flow fields near their uniform equilibrium values. The governing equations are reduced to the linear system of ordinary differential equations. The system is rigorously solved, and the eigenvalues and eigenvectors are found. The interface stability and the structure of the flow fields are fully investigated in a broad parameter regime. We have applied our general theoretical framework to the classical cases of the accelerated Landau-Darrieus instability and the Rayleigh-Taylor instability. We have reproduced the classical results and have found some features of the accelerated LD and RT dynamics that have not been discussed before. A detailed comparative study is conducted of the accelerated conservative dynamics with the accelerated Landau-Darrieus and the Rayleigh-Taylor dynamics. The properties of the interfacial accelerated conservative dynamics are found, which have not been identified earlier, Eqs. (1)-(11); Tables I-IV; Figs. $1-12$.

We have identified the new hydrodynamic instability that develops only when the acceleration magnitude $g$ exceeds a critical threshold $g_{c r}=k V_{h}^{2}\left(\rho_{h} / \rho_{l}\right)\left(\rho_{h}-\rho_{l}\right) /$ $\left(\rho_{h}+\rho_{l}\right)$. The accelerated dynamics is fully conservative. It conserves mass, momentum, and energy in the fluid bulk and their fluxes at the interface. It has potential velocity fields in the fluid bulk and is shear-free at the interface. For the accelerated conservative dynamics, the flow stability depends on the interplay of gravity and inertia, i.e., the gravity and the reactive force. For weak accelerations with gravity values smaller than a threshold, $g<g_{c r}$, the dynamics is stabilized by the inertial effect and the reactive force, and the flow fields experience stable oscillations. For strong acceleration, with large gravity values, $g>g_{c r}$, the gravitational effect dominates and gravity destabilizes the flow, and the instability develops, Eqs. (1)-(11); Tables I-IV; Figs. $1-12$.

The quantitative, qualitative, and formal properties of the unstable conservative dynamics depart from those of the accelerated Landau-Darrieus and the Rayleigh-Taylor dynamics. 3,4,20,23,29-31 For a finite density ratio and strong accelerations, this new instability grows faster than the accelerated LDI and RTI; for weak acceleration, the LDI has the largest growth-rate. For the unstable accelerated conservative dynamics, the flow is potential in the fluids' bulk, similarly to the RTI and in contrast to the accelerated LDI; it is shear-free at the interface, similarly to the LDI and in contrast to the RTI. The accelerated conservative dynamics is non-degenerate, in contrast to the LDI and RTI. The degeneracy suggests a singular (ill-posed) character of RT and LD dynamics, Eqs. (1)-(11); Tables I-IV; Figs. 1-12.

For the cases of the unstable accelerated conservative dynamics, accelerated LDI, and the RTI, the interface is unstable because the acceleration is directed from the heavy 
to the light fluid, and the initially planar interface is in a state of an unstable equilibrium corresponding to the maximum of potential energy of the fluids' system. Yet, the dynamics of the perturbed interface is highly sensitive to the boundary conditions at the interface, as it is unambiguously found in our results Eqs. (1)-(11); Tables I-IV; Figs. 1-12. For instance, for the flow fields, the accelerated conservative dynamics conserves at the interface the perturbed fluxes of mass, of normal and tangential components momentum and of energy; accelerated Landau-Darrieus dynamics conserves the perturbed fluxes of mass and of normal and tangential components of momentum and has zero perturbed mass flux; the Rayleigh-Taylor dynamics conserves the perturbed fluxes of mass and normal momentum and has zero perturbed mass flux at the interface, Eqs. (1)-(11); Tables I-III; Figs. 1-12.

It is well known that the presence of gradients of pressure and density in a fluid flow may lead to baroclinic vorticity production. ${ }^{3}$ This may happen when the pressure and density are continuous functions of the coordinates and time, and when these gradients are misaligned. ${ }^{3}$ When the fluid flow has a discontinuity (a front or an interface), a more cautious consideration is required, Eqs. (2a), (2d), and $(2 \mathrm{e}) .{ }^{3}$ On the side of mathematics, the boundary value should be solved, with the boundary conditions at the fluid interface and at the outside boundaries of the domain Eqs. (2a) and (3). ${ }^{3}$ On the side of physics, in the presence of an acceleration (a "strong pressure gradient") and an interface (a "strong density gradient"), the non-equilibrium perturbed flow usually has effectively no motion away from the interface and intense motion of the fluids near the interface, ${ }^{1,2,4,7-15,24-34,36}$ Figs. 3(a), 7(a), and 10. As such, the dynamics is set by the fluxes of mass, momentum and energy at the interface. The "interfacial" nature of the non-equilibrium dynamics is observed in experiments. ${ }^{11,13,36}$ A strong acceleration may even lead to the appearance of an interface in a flow with initially continuous density field. ${ }^{11,13,36}$

In this work, we have developed and applied the general matrix method to solve the boundary value problems Eqs. (2), (6), (9), and (10). We have found that indeed the nonequilibrium perturbed flow has effectively no motion away from the interface and intense motion of the fluids near the interface, and the latter is highly sensitive to the boundary conditions, Figs. 1-12. In particular, in the accelerated conservative dynamics and accelerated Landau-Darrieus dynamics, the tangential component of velocity is continuous at the interface, and the flow is shear-free at the interface, Eqs. (6a) and (9a); Figs. 3(a), 3(b), and 7(a). In the accelerated conservative dynamics, the perturbed velocity field is potential in the bulk, Fig. 3(a). In the accelerated Landau-Darrieus dynamics, the perturbed velocity field has the vortical field in the bulk of the light fluid, achieving its extreme values at the interfaces and decaying away from the interface, Fig. 8(a). In Rayleigh-Taylor dynamics, the velocity fields are potential in the bulk, and the vortical structures appear at the interface since the tangential velocity component is discontinuous at the interface, Fig. 10.

To better understand a transition from a flow with a discontinuity to a flow with continuously changing flow fields, further investigations are required to systematically account for non-ideal and compressible effects and a finite thickness of the interface, to be studied in future research.

For the accelerated conservative dynamics, the interplay of the inertial stabilization mechanism and the gravitational destabilization mechanism results in the appearance of the critical length-scale $\lambda_{c r}=2 \pi / k_{c r}$ at which the interface becomes stable and the length-scale $\lambda_{\max }=2 \pi / k_{\max }$ corresponding to the fastest growing mode in the unstable regime, with $k_{c r} / k_{\max }=2$ and $\lambda_{\max } / \lambda_{c r}=2$. For $g>g_{c r}$, the values $k_{c r(\max )}$ and $\lambda_{c r(\max )}$ are finite, with $k_{c r(\max )} \sim g / V_{h}^{2}$ and $\lambda_{c r(\max )} \sim V_{h}^{2} / g$. For accelerated Landau-Darrieus and Rayleigh-Taylor dynamics, the inertial stabilization mechanism is absent, and the values approach $k_{c r(\max )} /\left(g / V_{h}^{2}\right) \rightarrow$ $\infty$ and $\lambda_{c r \text { (max })} /\left(V_{h}^{2} / g\right) \rightarrow 0$. For the RTI, $V_{h}$ is understood as some velocity scale.

Our results suggest that the conservative dynamics can be stable even for ideal incompressible fluids when the gravity value is smaller than a threshold, similarly to ablative RTI and RMI in plasmas. ${ }^{14-16,25-28}$ In the unstable regime, depending on the gravity value and the density ratio, the conservative instability can grow faster or slower than the accelerated LDI and the RTI; it is the fastest in the extreme regimes of strong accelerations, Fig. 11, occurring, e.g., in HEDP. $^{6-8}$

Our results indicate a need in further experimental and numerical studies of the interface dynamics. Numerical modeling of unstable fluid interfaces is a challenge because the simulations are required to track the interface, to capture small scales dissipative processes, and to use the highly accurate numerical methods and massive computations. $1,11,12$ Existing numerical approaches usually apply diffusive approximation for modeling interfaces with the interfacial mass flux, and work well for flows with smoothly changing of flow fields. ${ }^{1}$ New developments may help to accurately model the unstable interface with sharply changing flow fields, including the Lagrangian and Eulerian methods. ${ }^{1,11,12}$

Our results suggest for experiments and simulations the following studies of the conservative dynamics with the growth-rate $\Omega=k V_{h} \sqrt{\left(\rho_{h} / \rho_{l}\right)\left(g / g_{c r}-1\right)}$. For fixed $g, V_{h},\left(\rho_{h} / \rho_{l}\right)$ one may vary perturbation wavelength and observe interface stabilization at $k_{c r}=\left(g / V_{h}^{2}\right)\left(\rho_{l} / \rho_{h}\right)\left(\rho_{h}\right.$ $\left.+\rho_{l}\right) /\left(\rho_{h}-\rho_{l}\right)$ and the fastest growing instability at $k_{\max }=k_{c r} / 2$. For fixed $V_{h},\left(\rho_{h} / \rho_{l}\right), \lambda$, one may vary $g$ and observe stable oscillations of the interface with $\Omega$ $=i k V_{h} \sqrt{\rho_{h} / \rho_{l}}$ for $g \ll g_{c r}$, and the unstable growth of the perturbations with $\Omega=k V_{h} \sqrt{\left(\rho_{h} / \rho_{l}\right) g / g_{c r}}$ for $g \gg g_{c r}$. For fixed $g,\left(\rho_{h} / \rho_{l}\right), \lambda$, one may vary $V_{h}$ and observe stable oscillations for large $V_{h}$ and unstable perturbations growth for small $V_{h}$. While these properties agree qualitatively with observations, in realistic environments, the material dynamics is usually accompanied by dissipation, diffusion, compressibility, radiation transport, stratification, surface tension, non-local forces, and other effects. ${ }^{1,6-19,25-28,36}$ The general approach that we have developed can be extended to systematically incorporate these effects, to analyze the interplay of the interface stability with the structure of vector and scalar flow fields, and to elaborate a unified theoretical framework for studies of interfacial dynamics in a broad range of processes including the ablative RTI and RMI in 
fusion plasmas, the dynamics of reactive and super-critical fluids, and the D'yakov-Kontorovich instability of the shock waves. ${ }^{1-33}$ We address these studies to future research.

Existing experimental and numerical studies of the interface stability are focused on the growth of the perturbation amplitude. ${ }^{1,6,7,12}$ Our analysis derives the amplitude growthrate, and finds that the flow dynamics is highly sensitive to the interfacial boundary conditions. We also find that the new diagnostics can help to grasp the properties of interfacial dynamics. In particular, according to our results, by measuring at macroscopic scales the flow fields in the bulk and at the interface as well as the interface morphology, one can capture the transport properties at microscopic scales at the interface, Eqs. (1)-(11); Tables I-IV; Figs. 1-12. This information is especially important for systems where experimental data are a challenge to obtain, including fusion, supernovae, and scramjets. ${ }^{1-36}$

\section{ACKNOWLEDGMENTS}

This work was supported by the University of Western Australia (AUS) via the project grant 10101047, the National Science Foundation (USA) via the award 1404449, the Japan Society for Promotion of Science (Japan) via the award S11140, the Caltech SURF program (USA) via the Toni and Bob Perpall SURF Fellowship in 2017, the Department of Energy (USA) via the award DE-SC0017710. The authors contributed to the work as follows: SIA designed research; DVI and SIA performed research; DVI, WAG, and SIA analyzed data; DVI, YF, WAG, and SIA discussed results; and DVI, WAG, and SIA wrote the paper.

${ }^{1}$ S. I. Abarzhi, S. Gauthier, and K. R. Sreenivasan, Philos. Trans. R. Soc. A 371, 20130268 (2013).

${ }^{2}$ Y. B. Zeldovich and Y. P. Raizer, Physics of Shock Waves and HighTemperature Hydrodynamic Phenomena, 2nd ed. (Dover, New York, 2002).

${ }^{3}$ L. D. Landau and E. M. Lifshitz, Course of Theoretical Physics VI Fluid Mechanics (Pergamon Press, New York, 1987).

${ }^{4}$ S. I. Abarzhi, Philos. Trans. R. Soc. A 368, 1809 (2010).

${ }^{5}$ W. A. Goddard, A. van Duin, K. Chenoweth, M.-J. Cheng, S. Pudar, J. Oxgaard, B. Merinov, Y. H. Jang, and P. Persson, Top. Catal. 38, 93 (2006).

${ }^{6}$ B. A. Remington, R. P. Drake, H. Takabe, and W. D. Arnett, Phys. Plasmas 7, 1641 (2000).
${ }^{7}$ S. E. Bodner, D. G. Colombat, J. H. Gardner, R. H. Lehmberg, R. L. McCrory, W. Seka, C. P. Verdon, J. P. Knauer, B. B. Afeyan, and H. T. Powell, Phys. Plasmas 5, 1901 (1998).

${ }^{8} \mathrm{D}$. Arnett, Supernovae and Nucleosynthesis: An Investigation of the History of Matter from the Big Bang to the Present (Princeton University Press, Princeton, NJ, 1996).

${ }^{9}$ V. Zhakhovsky, M. Budzevich, N. Inogamov, I. Oleynik, and C. White, Phys. Rev. Lett. 107, 135502 (2011).

${ }^{10}$ A. Prosperetti and M. S. Plesset, Phys. Fluids 27, 1590 (1984).

${ }^{11}$ S. I. Anisimov, R. P. Drake, S. Gauthier, E. E. Meshkov, and S. I. Abarzhi, Philos. Trans. R. Soc. A 371, 20130266 (2013).

${ }^{12}$ K. Kadau, J. L. Barber, T. C. Germann, B. L. Holian, and B. J. Alder, Philos. Trans. R. Soc. 368, 1547 (2010).

${ }^{13}$ E. E. Meshkov, Studies of Hydrodynamic Instabilities in Laboratory Experiments (FGUP 'RFYC-VNIIEF', Sarov, 2006) (in Russian), ISBN: 5-9515-0069-9.

${ }^{14}$ H. J. Kull and S. I. Anisimov, Phys. Fluids 29, 2067-2075 (1986).

${ }^{15}$ O. A. Hurricane, D. A. Callahan, D. T. Casey, E. L. Dewald, T. R. Dittrich, T. Döppner, M. A. B. Garcia, D. E. Hinkel, L. F. B. Hopkins, P. Kervin et al., Phys. Plasmas 21, 056314 (2014).

${ }^{16}$ J. Sanz, Phys. Rev. Lett. 73, 2700 (1994).

${ }^{17}$ N. Peters, Turbulent Combustion (Cambridge University Press, 2000).

${ }^{18}$ P. K. Wu, K. A. Kirkendall, R. P. Fuller, and A. S. Nejad, J. Propul. Power 13, 64 (1997).

${ }^{19}$ W. D. Arnett, C. Meakin, and P. A. Young, Astrophys. J. 690, 1715 (2009).

${ }^{20}$ L. D. Landau, Acta Physicochim. URSS 19, 77 (1944).

${ }^{21}$ F. A. Williams, Ann. Rev. Fluid Mech. 3, 171 (1971).

${ }^{22}$ G. H. Markstein, J. Aeronaut. Sci. 18, 428 (1951).

${ }^{23}$ Y. B. Zeldovich, The Mathematical Theory of Combustion and Explosions (Springer, 1944, 1985), ISBN-10: 1461294398, ISBN-13: 978-1461294399.

${ }^{24}$ S. I. Abarzhi, Y. Fukumoto, and L. P. Kadanoff, Phys. Scr. 90, 018002 (2015).

${ }^{25}$ B. A. Remington, S. V. Weber, M. M. Marinak, S. W. Haan, J. D. Kilkenny, R. J. Wallace, and G. Dimonte, Phys. Plasmas 2, 241 (1995).

${ }^{26}$ H. Azechi, T. Sakaiya, S. Fujioka, Y. Tamari, K. Otani, K. Shigemori, M. Nakai, H. Shiraga, N. Miyanaga, and K. Mima, Phys. Rev. Lett. 98, 045002 (2007)

${ }^{27}$ K. Shigemori, H. Azechi, M. Nakai, M. Honda, K. Meguro, N. Miyanaga, H. Takabe, and K. Mima, Phys. Rev. Lett. 78, 250 (1997).

${ }^{28}$ Y. Aglitskiy, A. L. Velikovich, M. Karasik, N. Metzler, S. T. Zalesak, A. J. Schmitt, L. Phillips, J. H. Gardner, V. Serlin, J. L. Weaver, and S. P. Obenschain, Philos. Trans. R. Soc. A 368, 1739 (2010).

${ }^{29}$ L. Rayleigh, Proc. London Math. Soc. 14, 170 (1883).

${ }^{30}$ R. Davies and G. I. Taylor, Proc. R. Soc. London A 200, 375 (1950).

${ }^{31}$ S. I. Abarzhi, K. Nishihara, and R. Rosner, Phys. Rev. E 73, 036310 (2006).

${ }^{32}$ S. P. D’yakov, Zh. Exp. Teor. Fiz 33, 948 (1957).

${ }^{33}$ V. M. Kontorovich, Zh. Exp. Teor. Fiz 33, 1527 (1957).

${ }^{34}$ S. I. Abarzhi, D. V. Ilyin, W. A. Goddard, and S. I. Anisimov, "Interface dynamics: Mechanisms of stabilization and destabilization and structure of flow fields," Proc. Natl. Acad. Sci. U. S. A. (published online).

${ }^{35}$ D. V. Beklemishev, Course of Analytical Geometry and Linear Algebra (Nauka, Moscow, 1971).

${ }^{36}$ E. E. Meshkov, Philos. Trans. R. Soc. A 371, 20120288 (2013). 